Astrophysical Journal, Submitted

Preprint typeset using $\mathrm{LATEX}_{\mathrm{E}}$ style emulateapj v. 5/2/11

\title{
SUZAKU X-RAY OBSERVATIONS OF THE NEAREST NON-COOL CORE CLUSTER, ANTLIA: DYNAMICALLY YOUNG BUT WITH REMARKABLY RELAXED OUTSKIRTS
}

\author{
Ka-Wah Wong ${ }^{1,2}$, Jimmy A. Irwin ${ }^{3}$, Daniel R. Wik ${ }^{4,5}$, Ming Sun $^{6}$, Craig L. Sarazin ${ }^{7}$, Yutaka Fujita ${ }^{8}$, And Thomas \\ H. REIPRICH ${ }^{9}$, \\ Astrophysical Journal, submitted
}

\begin{abstract}
We present the results of seven Suzaku mosaic observations ( $>200 \mathrm{ks}$ in total) of the nearest non-cool core cluster, the Antlia Cluster (or Group), beyond its degree-scale virial radius in its eastern direction. The temperature is consistent with the scaled profiles of many other clusters. Its pressure follows the universal profile. The density slope in its outskirts is significantly steeper than that of the nearest cool core cluster (Virgo) with a similar temperature as Antlia, but shallower than those of the massive clusters. The entropy increases all the way out to $R_{200}$, which is consistent in value with the baseline model predicted by a gravity heating-only mechanism in the outskirts. Antlia is quite relaxed in this direction. However, the entropy inside $\sim R_{500}$ is significantly higher than the baseline model, similar to many other nearby low mass clusters or groups. The enclosed gas-mass fraction does not exceed the cosmic value out to $1.3 R_{200}$. Thus, there is no evidence of significant gas clumping, electron-ion non-equipartition, or departure from the hydrostatic equilibrium approximation that are suggested to explain the entropy and gas fraction anomalies found in the outskirts of some massive clusters. We also present scaling relations for the gas fraction $\left(f_{\text {gas,200 }}\right)$, entropy $\left(K_{200}\right)$, and temperature $\left(T_{500}\right)$ using 22 groups and clusters with published data in the literature. The enclosed baryon fraction at $R_{200}$ is broadly consistent with the cosmic value. The power law slope of the $K_{200}-T_{500}$ relation is $0.638 \pm 0.205$. The entropy deficit at $R_{200}$ cannot be fully accounted for by the bias or deviation in the gas fraction.
\end{abstract}

Subject headings: X-rays: galaxies: clusters - galaxies: clusters: intracluster medium - galaxies: groups: individual (the Antlia Cluster) — intergalactic medium - cosmology: large-scale structure of universe

\section{INTRODUCTION}

X-ray observations have shown that the study of the intracluster medium (ICM) can be used to test plasma physics under extreme conditions that cannot be achieved in terrestrial laboratories, as well as an important cosmological probe. However, the study of cosmology using clusters of galaxies relies heavily on the understanding of cluster physics. For precision cosmology, systematic uncertainties at even the percent level are quite significant. Even though present X-ray measurements of clusters for cosmological studies are limited to the inner regions ( $\lesssim R_{500}$ due to sensitivity limit: ${ }^{10}$ ), many X-ray observations and Sunyaev-Zel'dovich (SZ) observations

kw6k@email.virginia.edu

${ }^{1}$ Eureka Scientitic, Inc., 2452 Delmer Street Suite 100, Oakland, CA 94602-3017, USA

2 Department of Physics and Astronomy, Minnesota State University, Mankato, MN 56001, USA

3 Department of Physics and Astronomy, University of $\mathrm{Al}$ abama, Box 870324, Tuscaloosa, AL 35487, USA

4 Astrophysics Science Division, NASA/Goddard Space Flight Center, Greenbelt, MD 20771, USA

5 The Johns Hopkins University, Homewood Campus, Baltimore, MD 21218, USA

${ }^{6}$ Physics Department, University of Alabama in Huntsville, Huntsville, AL 35899, USA

7 Department of Astronomy, University of Virginia, P.O. Box 400325, Charlottesville, VA 22904-4325, USA

8 Department of Earth and Space Science, Graduate School of Science, Osaka University, Toyonaka, Osaka 560-0043, Japan

9 Argelander Institut für Astronomie, Universität Bonn, Auf dem Hügel 71, D-53121, Germany

$10 R_{\Delta}$ is the radius within which the mean total mass density of the cluster is $\Delta$ times the critical density of the universe. The have already extended to larger radii. Thus, a full understanding of the outer parts of clusters is essential.

Compared to the central regions, cluster envelopes were thought to be relatively simple because they are less subject to additional physics including cooling, and AGN feedback, and that the cluster outer regions might provide better cosmological probes. This appeared to be supported by earlier observational results with ROSAT that the X-ray surface brightness around $R_{200}$ is generally consistent with numerical simulations. However, more recent observations, primarily with Suzaku, have shown a number of unexpected results (see a recent review by Reiprich et al. 2013). For example, it has been suggested that the ICM near $R_{200}$ can be rather clumpy, which means the enclosed gas-mass fraction measured with X-ray can be biased high and may exceed the cosmic baryon fraction (e.g., Nagai \& Lau 2011; Simionescu et al. 2011); although Walker et al. (2012c) and Okabe et al. (2014) pointed out that clumpiness in the simulations by Nagai \& Lau (2011) becomes dominant only beyond $R_{200}$. The entropy near $R_{200}$ of some massive clusters measured with Suzaku appears to be significantly lower than the predictions from the gravity heating-only models (see, e.g., Walker et al. 2013, and references therein), although the ROSAT results are less significant (Eckert et al. 2013). This low entropy

virial radius $R_{\mathrm{vir}}$ is defined as a radius within which the cluster is virialized. For the Einstein-de Sitter universe, $R_{\text {vir }} \approx R_{200}$, while for the standard $\Lambda$ CDM Universe, $R_{\text {vir }} \approx R_{100}$. In this paper, we also call $R_{200}$ the virial radius since this definition is still widely used in the literature. 
may indicate that the collisional equilibrium of electrons and ions has not been achieved in the low density hot gas in cluster outskirts (e.g., Wong \& Sarazin 2009; Hoshino et al. 2010; Akamatsu et al. 2011; Wong et al. 2011; Avestruz et al. 2015), which may introduce biases in cosmological parameters (Wong et al. 2010). Pressure support from turbulence/bulk motions (e.g., Lau et al. 2009) or cosmic rays (Fujita et al. 2013) are also thought to be important around $R_{200}$, resulting in the breakdown of the hydrostatic equilibrium (HSE) approximation if only the thermal pressure is considered (e.g., Kawaharada et al. 2010; Ichikawa et al. 2013; Okabe et al. 2014). More than a dozen clusters have been studied with spatially and spectrally resolved gas profiles out to $\sim R_{200}$ to understand these anomalous effects (see again the review by Reiprich et al. 2013).

Mapping the nearest clusters with mosaic pointings to cover angular scales of order degrees has revealed the complex ICM structures in tremendous spatial detail. A direct comparison to the nearest clusters with different physical properties in our neighborhood is certainly important to understand the cluster outskirts. For example, the nearby massive cool core cluster, Perseus (distance $D=70 \mathrm{Mpc}, \quad R_{200}=1.8 \mathrm{Mpc}=1.4^{\circ}:$ Simionescu et al. 2011; Urban et al. 2014), shows significant bias in the gas fraction and entropy deficit near $R_{200}$, as well as strong azimuthal variations. The massive merging cluster, Coma $\left(D=100 \mathrm{Mpc}, R_{200} \sim 2 \mathrm{Mpc}=1.2^{\circ}\right.$ : Akamatsu et al. 2013; Simionescu et al. 2013), shows both a dynamical active environment with a merging group in one direction and a relaxed environment along the other directions.

The nearest cluster (or group), Virgo $(D=16 \mathrm{Mpc}$, $R_{200}=1 \mathrm{Mpc}=4^{\circ}$, which is also a cool core with a lower $\operatorname{mass}\left(M_{500} \approx 10^{14} M_{\odot}\right)$ and temperature $(\approx 2.3 \mathrm{keV})$ compared to the massive clusters $\left(M_{500} \gtrsim 5 \times 10^{14} M_{\odot}\right)$ mentioned above, has also been mapped out to $R_{200}$ (Urban et al. 2011; Simionescu et al. 2015). Low mass galaxy groups are in fact also very important to cosmological studies. Because of their high abundance, they contribute significantly to the SZ power spectrum at angular scales of $l \approx 3000$ that are sensitive to cosmological parameters (Trac et al. 2011). The previous XMMNewton result for Virgo suggests that its density profile near $R_{200}$ has a power law $\left(n_{e} \propto r^{-\alpha}\right)$ slope of $\alpha=1.2 \pm 0.2$, 四 which is significantly flatter than for massive cluster counterparts (e.g., $\alpha=1.7 \pm 0.2$ for Perseus, Urban et al. 2014; $\alpha=2.27 \pm 0.07$ for A1795, Bautz et al. 2009; and $\alpha=2-3$ for a stacked Chandra sample, Morandi et al. 2015). Therefore, it is natural to wonder whether non-cool core groups behave in the same manner or differently, compared with massive clusters or the Virgo cool core group; understanding this will have deep implications for both cluster studies and cosmology.

Sitting at a distance of $D=39.8 \mathrm{Mpc}$ (Cantiello et al. 2005), the Antlia Cluster is the nearest non-cool core cluster (or a group with similar size to the Virgo Cluster) and also the third closest galaxy cluster after the Fornax cool core cluster (group). X-ray observations suggest that the Antlia core is approximately isothermal $(k T \sim 2 \mathrm{keV})$ with no significant excess central

11 The errors for Virgo and Perseus have been converted to $90 \%$ confidence. brightness (Nakazawa et al. 2000). The X-ray emission is centered on the bright elliptical galaxy NGC 3268 and is elongated toward the southwest where there is a subgroup centered on another bright elliptical galaxy NGC 3258, indicating that the Antlia Cluster is accreting along this direction (Figure 1) see also Pedersen et al. 1997; Nakazawa et al. 2000). Optical observations suggest that Antlia is dynamically younger than Virgo and Fornax, and the large-scale filament structure of the galaxies are along the northwest and southeast directions. (Ferguson \& Sandage 1990; Smith Castelli et al. 2008; Hess et al. 2015).

Here, we report the results from mosaic Suzaku observations of Antlia to the east direction out to $1.3 R_{200}$. Compared to studying cool core clusters/groups, the non-cool core Antlia is less subject to systematic uncertainty due to the telescope scattering of X-ray photons from the bright center to the outer regions, which are of greatest interest here. The direction was chosen so that it is away from the accreting filament direction where substructures induced by accretion can disturb the global properties significantly. Thus, the eastern direction should be more relaxed compared to the filament directions, as we will also show in Section 9.1 below. It was also chosen to avoid many of the point sources and the high background seen on the ROSAT image. We made use of the Chandra and XMM-Newton data to minimize the uncertainty due to point sources unresolved by Suzaku (Miller et al. 2012). We also compared our results with ROSAT data to ensure that the soft X-ray emission determined with Suzaku was robust (see, e.g., Eckert et al. 2011).

With the growing number of clusters and groups measured out to $R_{200}$, it is possible to study the scaling relations between the gas fraction $\left(f_{\text {gas,200 }}\right)$, entropy $\left(K_{200}\right)$, and temperature where the gas fraction and entropy are of great interest to constrain cosmological parameters and to understand the thermodynamic history of cluster or group formations, respectively (see, e.g., Sun et al. 2009; ; Pratt et al. 2010). Thus, we compiled the observational data of 22 groups and clusters from the literature to study the $f_{\text {gas } 200}-T_{500}$ and $K_{200}-T_{500}$ relations out to $R_{200}$.

The paper is organized as follows. Section 2 describes the X-ray observations and data analysis. The following sections describe the observational results, including the surface brightness (Section 3); temperature (Section 4); density, pressure, and entropy (Section 5); mass (Section 6); and equilibration timescale (Section 17) profiles of the hot gas. We present the $f_{\text {gas }, 200}-T_{500}$ and $K_{200^{-}}$ $T_{500}$ relations in Section 8 We compare Antlia to other galaxy groups and massive clusters and discuss the implications in Section 9. We summarize our conclusions in Section 10. Appendix \$ presents the density deprojection methods. Systematic uncertainties in spectral modeling are addressed in detail in Appendix B.

At a distance of $39.8 \mathrm{Mpc}$, the angular scale of the Antlia Cluster is $11.6 \mathrm{kpc} / 1^{\prime}$. We assume $H_{0}=70 \mathrm{~km} \mathrm{~s}^{-1} \mathrm{Mpc}^{-1}, \Omega_{M}=0.3$, and $\Omega_{\Lambda}=0.7$. The average X-ray temperature of Antlia beyond the core region is determined to be $T_{X}=1.54 \mathrm{keV}$ (Section 44). We adopted the scale radius of $R_{500}=591 h_{70}^{-1} \mathrm{kpc}\left(T_{X} / 1.54 \mathrm{keV}\right)^{0.55}=51^{\prime}$ inferred from 

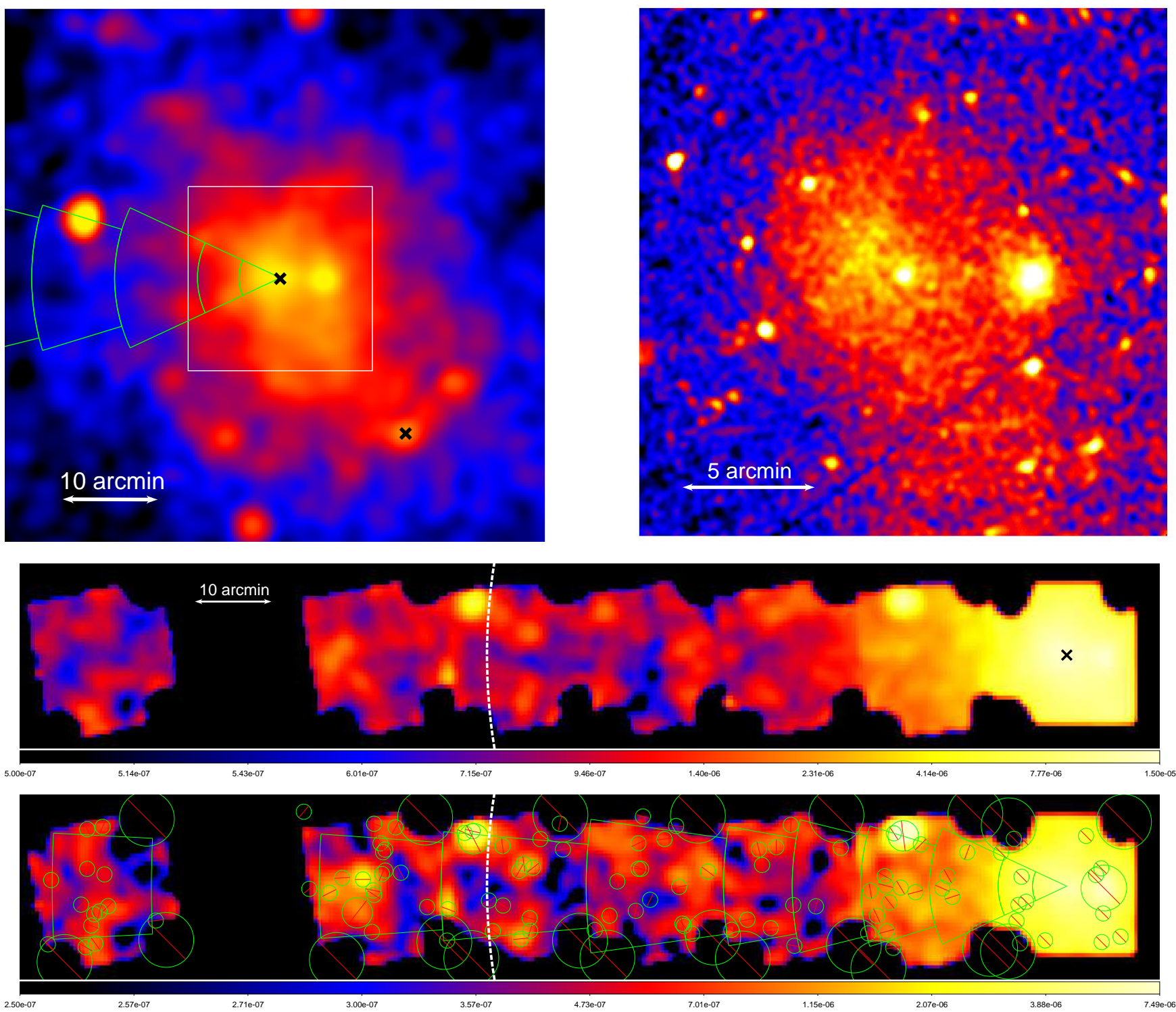

Fig. 1. - Smoothed ROSAT PSPC image of the central regions of the Antlia Cluster in the 0.5-2 keV band is shown in the upper left. North is up, while east is left. The color represents the X-ray intensity from high (yellow-red) to low (dark blue). The upper and lower black crosses indicate the locations of NGC 3268 and NGC 3258, respectively. The X-ray peak about $5^{\prime}$ west of NGC 3268 is probably a background cluster (Section 2.2). The innermost four spectral extraction areas of the Suzaku data analysis are shown in green pie regions. The white square indicates the field of view of the smoothed XMM-Newton MOS2 image (Obs. ID: 0691950201: PI: E. T. Million) in the 0.5-2 keV band shown on the upper right panel. Also shown are the exposure corrected, background subtracted, and smoothed soft-band (0.6-2.0 keV: middle) and hard-band (2.0-7.0 keV: bottom) Suzaku images of Antlia with an image pixel binning size $=0.5$. Both images were smoothed with a Gaussian kernel of FWHM $\approx 1^{\prime}$. The color bars show the surface brightness in units of count s $\mathrm{sm}^{-2} \operatorname{arcmin}^{-2}$. The seven Suzaku pointings from the right to the left are E0, E1, E2, E3, E4, E5, and the local background field EB. The dashed white arc on the middle image has a radius of $R_{200}=887 \mathrm{kpc}=76^{\prime}$ centered on the peak of the extended emission (black cross). The removed point sources (or compact structures) and the calibration sources (at field corners) are shown in solid green circles (with a red line across) on the bottom image. The spectral extraction areas are shown in solid green pie regions on the bottom image. The unresolved diffuse emission in the soft-band image is dominated by hot gas within $\lesssim 30^{\prime}$ while the hard-band image is dominated by CXB beyond $\gtrsim 20^{\prime}$. 
the X-ray scaling relation of Sun et al. (2009). For low mass galaxy groups, the concentration parameter $c_{200}$ of the NFW dark matter model is about six, and therefore $R_{200} \approx 1.5 R_{500}=887 \mathrm{kpc}=76^{\prime}$ (e.g., Figure 4 in Yang et al. 2009). Errors are given at 90\% confidence level in this paper unless otherwise specified.

\section{X-RAY OBSERVATIONS AND SPECTRAL ANALYSIS}

\subsection{Data Reduction}

The Antlia Cluster (Figure 1) was observed with Suzaku at the center in 2008 (PI: T. Kitaguchi), and also along the east direction for six pointings out to a radius of $\sim 135^{\prime}$ in 2012 (PI: K.-W. Wong). The central pointing (E0) was observed for $66 \mathrm{ks}$. Each of the next three pointings from the center (E1-E3) were observed for $23-26 \mathrm{ks}$, and the outermost three pointings (E4, E5, and EB) were each observed for $46-47 \mathrm{ks}$. These seven observations are listed in Table 1.

The Suzaku XIS0, XIS1, and XIS3 data were reduced using the HEAs oft package version $6.15^{12}$ and the Suzaku CALDB version 20140203. All the data were reprocessed using the FTOOLS aepipeline script in the HEAsoft package. All the standard screening criteria were applied I3 In addition, we selected data with the geomagnetic cut-off rigidity $\mathrm{COR} 2>6 \mathrm{GV}$ to reduce the particle-induced non-X-ray background (NXB), and removed events in regions illuminated by the XIS calibration sources. For the XIS1 data observed in 2012, the increase of charge injection enhanced the NXB. To reduce the NXB, we have also followed the recommended procedure 14 in the pipeline processing. The data with $3 \times 3$ and $5 \times 5$ editing modes for each pointing were merged. We have examined the $12-14 \mathrm{keV}$ light curves of the screened data and did not find any flares in the NXB.

For each pointing, we constructed light curves and removed data with count rates that deviated by $>3 \sigma$ from the mean to avoid potential flares due to solar wind charge exchange (SWCX) or astrophysical sources, among others. Two energy bands of 0.5-8.0 and 0.4$1.0 \mathrm{keV}$ were used, with the latter energy band chosen to check for contamination due to SWCX. After that, we visually inspected each light curve and found that in the observation E4, the count rate of the second half of the observation in $0.4-1.0 \mathrm{keV}$ was about twice as high as the first half of the observation. The enhanced solar proton flux by up to a factor of seven measured by the Advanced Composition Explorer ( $A C E$ ) during this observation suggests an increase in SWCX, and therefore we have also removed the second half of this observation. The cleaned effective exposure times are listed in Table 1 .

NXB files for each detector and observation were created according to the cut-off rigidity weighting using the FTOOLS xisnxbgen script with the same filtering criteria mentioned above. We selected NXB data within \pm 300 days of each observation. These backgrounds were subtracted from the image and spectral analysis below.

\subsection{Point Source Removal}

\footnotetext{
12 http://heasarc.gsfc.nasa.gov/docs/software/lheasoft/

$13 \mathrm{http}$ ///heasarc.nasa.gov/docs/suzaku/processing/criteria_xis.html

14 http://heasarc.gsfc.nasa.gov/docs/suzaku/analysis/abc/ node8.html
}

TABLE 1

OBSERVATIONS

\begin{tabular}{cclcl}
\hline \hline Name & Obs. ID & Obs. Date & Exp. $(\mathrm{ks})^{\mathrm{a}}$ & $n_{\mathrm{H}}{ }^{\mathrm{b}}$ \\
\hline Suzaku & & & & \\
\hline Antlia E0 & 802035010 & 2007 Nov 19 & 55 & 8.84 \\
Antlia E1 & 807066010 & 2012 Jun 13 & 20 & 8.90 \\
Antlia E2 & 807067010 & 2012 Jun 14 & 21 & 9.00 \\
Antlia E3 & 807068010 & 2012 Jun 15 & 19 & 9.07 \\
Antlia E4 & 807069010 & 2012 Jun 16 & 17 & 9.14 \\
Antlia E5 & 807070010 & 2012 Jun 17 & 39 & 9.09 \\
Antlia EB & 807071010 & 2012 Jun 18 & 38 & 8.17 \\
\hline & & & & \\
XM-Newton & & & & \\
\hline Antlia E0 & 0604890101 & 2010 Jan 03 & $52 / 48$ & \\
\hline Chandra & & & 7 & \\
Antlia E1 & 15090 & 2013 Nov 20 & 7 & \\
Antlia E2 & 15089 & 2013 Nov 22 & 7 & \\
Antlia E3 & 15088 & 2013 Jul 02 & 7 & \\
Antlia E4 & 15086 & 2013 Nov 04 & 7 & \\
Antlia E5 & 15085 & 2013 Apr 05 & 7 & \\
Antlia EB & 15087 & 2013 Nov 04 & 7 & \\
\hline
\end{tabular}

a Effective exposure time after cleaning. The XMM-Newton exposures are for MOS/PN.

b Hydrogen column density in units of $10^{20} \mathrm{~cm}^{-2}$ Willingale et al. 2013).

The bottom panel of Figure 1 shows the hard-band (2$7 \mathrm{keV}$ ) Suzaku image, with green circles indicating the XIS calibration sources and other contamination sources removed during the analysis.

The contamination sources were detected as follows: The central region (E0) of the Antlia was observed with XMM-Newton in 2010 (ObsID 0604890101, PI: M. Machacek, Table 1). Because the ICM emission from the central region of the Antlia Cluster is bright enough and background contamination from point sources or compact structures is not important, we simply took the archival XMM-Newton full EPIC image in $0.2-12 \mathrm{keV}$ and detected sources using the CIAO wavdetect. We examined the brightest 20 sources, where the flux is above the Chandra detection limit of $\approx 1.4 \times 10^{-14} \mathrm{erg} \mathrm{s}^{-1} \mathrm{~cm}^{-2}$ in $2-10 \mathrm{keV}$ (see below), for potential source removal in the Suzaku data analysis. One of them is located at the center of the Antlia Cluster and is the peak of the ICM emission; therefore, it should not be excluded from the data analysis. Four other sources are outside the Suzaku field of view (FOV). One source (10:29:36.725, $-35: 19: 36.38$ ) detected is extended and is about $5^{\prime}$ away from the center of the Antlia Cluster to the west direction. Spectral analysis indicates that the emission is thermal. The location of its Fe K line suggests that it is probably a background cluster located at a high redshift of $z=0.4$. It has a temperature of $4 \mathrm{keV}$ and $R_{500} \approx 0.9 \mathrm{Mpc} \sqrt{T_{X} / 4 \mathrm{keV}} \approx 3^{\prime}$. A circular region of $3^{\prime}$ in the radius of this background cluster candidate is excluded from the Suzaku data analysis. We exclude all other sources using circular regions of $1^{\prime}$ in radius. We visually inspected the Suzaku E0 data and did not find any source structure that was not detected by the $X M M$ Newton observations.

The fainter outer regions, especially near the virial radius, could be more subject to point source contaminations. We obtained six Chandra ACIS-I observations in 2013 (PI: K.-W. Wong, Table 1), centered on each of the six Suzaku pointings away from the clus- 
ter center. Each Chandra pointing was observed for 7 ks. The Chandra ACIS-I observations cover most of the Suzaku XIS observed regions due to their similar FOVs. We created images with 0 "' 492 pixels in a broad energy band $(0.1-10 \mathrm{keV})$, and detected point sources with CIAO wavdetect. Sources detected with $>3 \sigma$ were removed during the Suzaku data analysis. The detection limit is about $1.4 \times 10^{-14} \mathrm{erg} \mathrm{cm}^{-2} \mathrm{~s}^{-1}$ in $2-10 \mathrm{keV}$. We also visually inspected Suzaku images and excluded obvious bright point-like sources missed by the CIAO wavdetect script. Most of these sources were removed using a circular region size of $1^{\prime}$ in radius, with a few exceptions where larger sizes $\left(1^{\prime} .5\right.$ or $\left.2^{\prime}\right)$ were used for those with emission clearly extending larger than $1^{\prime}$ in radius.

The half-power diameter (HPD) of Suzaku is about $2^{\prime}$ and therefore about half the photons from point sources fell outside our point source removal regions, and some of them entered the spectral extraction regions as residual signals. We took into account the residual contributions in the spectral analysis (Section 2.3.2). We note that the $X M M-N e w t o n$ or Chandra observations were taken close in time, but not simultaneously with the Suzaku observations. Because these residual signals make a relatively small contribution to the spectra (Section 3), the potential time variation of the integrated flux of the point sources should not be important.

\subsection{Spectral Analysis}

For each Suzaku XIS detector (XIS0, XIS1, and XIS3), we extracted spectra in pie regions centered on the central peak of the extended X-ray emission. These pie regions, as well as the contamination exclusion regions, are shown in the lower panel of Figure 1. The redistribution functions (RMF files) for each spectrum were generated using the FTOOLS xisrmfgen script. The corresponding ancillary response functions (ARF files) were generated using the FTOOLS xissimarfgen script. Because the spatial distributions of the ICM and background are different, different ARF files are required for spectral fitting.

When generating the ARF files for the ICM component for regions within $36^{\prime}$, we used the X-ray surface profile of the Antlia Cluster as the input for the xissimarfgen. For the inner $9^{\prime}$, the surface profile was modeled as a $\beta$-model fitted to the XMM-Newton data. The region beyond $9^{\prime}$ was modeled using the Suzaku data with the following iterative procedure. We first generated the ARF files using the standard $20^{\prime}$ radius uniform surface brightness source. We then determined the "initial" ICM emission measure using these uniform ARF files (using the same fitting procedure as the final spectral analysis below). We then fitted a double $\beta$-model to the "initial" ICM emission measure profile. This double $\beta$-model was used to calculate the "initial" surface brightness profile for the proper ARF files generation. We found that the final ICM emission measure determined using the proper ARF files is generally different from this "initial" emission measure by $<10 \%$ and smaller than the emission profile error, justifying the iterative procedure (see also Bautz et al. 2009). For regions beyond $\sim 20^{\prime}-30^{\prime}$, the systematic uncertainty in the stray light calibration is very large, and therefore the ARF files generated using the cluster model input may not be reliable (E. D. Miller 2015, private communication; see also Section 6.2.6 of the Suzaku Technical Description [15). Using XISSIM, we found that the cluster stray light beyond $\sim 36^{\prime}$ is less than $\sim 6 \%$ of the NXB. Therefore, the standard $20^{\prime}$ radius uniform surface brightness source was used as the input for the xissimarfgen for regions beyond $36^{\prime}$. We checked that the differences of best-fit hot gas parameters are less than $2 \%$ when using the ARF files generated by the two methods for the regions between $27^{\prime}$ and $36^{\prime}$. The difference should be negligible for regions beyond that, justifying the use of uniform surface brightness as the input. For the background model fitting, the standard uniform ARF files were used in all regions.

As mentioned in Section 2.1. NXB spectra with proper cut-off-rigidity weighting were extracted from nightearth data using the FTOOLS xisnxbgen. For the six observations taken in 2012 (E1-E5 and EB), we followed the standard procedures in the Suzaku Data Reduction Guide to mitigate the increase in NXB for XIS1 due to the $6 \mathrm{keV}$ charge injection 16 Because of the similar responses, the XIS0 and XIS3 (FI) source and background spectra and the response files were combined. We analyzed spectra between 0.6 and $7.0 \mathrm{keV}$. The lower energy limit was chosen to minimize the Suzaku calibration uncertainties below $\sim 0.6 \mathrm{keV}$, while the upper energy limit is chosen due to the dominating NXB above $\sim 7 \mathrm{keV}$. All the spectra were grouped with a minimum of one count per bin, and were first fitted using the c-statistic in the Xray Spectral Fitting Package (XSPEC) ${ }^{17}$ We then assessed the best-fit parameters (as median) and uncertainties using the MCMC method in XSPEC.

\subsubsection{ICM Spectral Model}

The ICM emission was modeled as an absorbed, optically thin thermal plasma model PHABS*APEC using the atomic database AtomDB version 2.0.2 (Smith et al. 2001; Foster et al. 2012). For each pointing, the absorption was fixed to the Galactic value (Table 1) determined by Willingale et al. (2013). These values are generally $\sim 25-$ $30 \%$ higher than those determined by Kalberla et al. (2005). We have included this uncertainty by varying the Galactic values by $\pm 30 \%$ in our analysis (Appendix B). The redshift is fixed at $z=0.00933$ (NASA/IPAC Extragalactic Database: NED). We adopted the solar abundance table from Asplund et al. (2009, hereafter aspl) for the ICM model, as well as the background model below. We have also assessed the abundance tables from Anders \& Grevesse (1989, hereafter angr), Grevesse \& Sauval (1998, hereafter grsa), and Lodders (2003, hereafter lodd), which are widely used in the literature. These systematic uncertainties have been taken into account in our analysis (Appendix B). The metallicity was thawed for the inner regions within $18^{\prime}$. For regions beyond that, where metallicity cannot be constrained, we fixed it to the lowest value of $0.15 Z_{\odot}$ obtained in the inner region as our nominal model. We have also fixed the metallicity to the maximum value of $0.4 Z_{\odot}$ determined at the center, and included this systematic uncertainty in our analysis (Appendix B).

We carried out both projected and deprojected spec-

\footnotetext{
15 http://heasarc.gsfc.nasa.gov/docs/suzaku/prop_tools/suzaku_td/ suzaku_td.html

16 http://heasarc.gsfc.nasa.gov/docs/suzaku/analysis/abc/abc.html

17 http://heasarc.nasa.gov/xanadu/xspec/
} 
tral analysis for the ICM component. For the projected spectral analysis, a single PHABS*APEC model was used for the ICM component for each spectral region. For the deprojected spectral analysis, the mixed model of PROJCT* (PHABS*APEC) was used instead. We assume the ICM to be spherically symmetric for the PROJCT model, and the optional keywords for position angles have been properly adjusted to account for the partial annular spectral regions.

\subsubsection{Background Model}

The outermost pointing beyond the virial radius (EB) was chosen to be a local background. The NXB is about $20(35) \%$ of the total counts for either the XIS FI and BI detectors in the 0.6-2.0 (0.6-7.0) keV band in the spectral extraction region of this pointing; it is subtracted (from all the other data as well) during the analysis. Varying the NXB by $\pm 5 \%$ (90\% confidence of systematic uncertainty; Tawa et al. 2008) does not change the results of the paper qualitatively (Appendix B). This systematic error has been included in our data results.

The residual X-ray background is primarily the residual signals from the removed point sources, the remaining unresolved cosmic X-ray background (CXB), Galactic Xray foreground (GXB), and potential contamination of line emission from SWCX.

To take into account the residual signals from the removed point sources, we first fitted the Chandra and $X M M$-Newton spectra for the removed point sources using an absorbed power law model with all the parameters thawed. Using the xissim script, we simulated Suzaku event files of these removed point sources for each pointing based on the spectra and fluxes determined. The simulation exposures were set to $500 \mathrm{ks}$ to improve the statistics. The fluxes of the residual signals were then determined from the simulated data. The contributions were modeled as the best-fit absorbed power law model (but to the residual flux) in the spectra analysis.

The CXB was modeled as a power law with a fixed photon index of 1.4 (Kushino et al. 2002) and a thawed normalization. Thawing the photon index gives a value of 1.39 (1.37) for the (de-)projection spectral analyisis, and this systematic uncertainty was taken into account in our results (Appendix B). We determined that the surface brightness of this residual $\mathrm{CXB}$ is generally $S_{\mathrm{CXB}, \text { Suzaku }}=(1.2-1.8) \times 10^{-11} \mathrm{erg} \mathrm{cm}^{-2} \mathrm{~s}^{-1} \mathrm{deg}^{-2}$ in $2-10 \mathrm{keV}$ in the outer regions beyond $27^{\prime}$. Since our point source detection limit is about $1.4 \times$ $10^{-14} \mathrm{erg} \mathrm{cm}^{-2} \mathrm{~s}^{-1}$ in this band, the expected level of the unresolved CXB is estimated to be $S_{\mathrm{CXB} \text {,expect }} \approx 1.3 \times$ $10^{-11} \mathrm{erg} \mathrm{cm}^{-2} \mathrm{~s}^{-1} \mathrm{deg}^{-2}$ with a $1 \sigma$ cosmic variance of $\sigma_{S} \approx 1.1 \times 10^{-12}\left(\Omega / 150 \operatorname{arcmin}^{2}\right)^{-0.5} \mathrm{erg} \mathrm{cm}^{-2} \mathrm{~s}^{-1} \mathrm{deg}^{-2}$ (Moretti et al. 2003; Moretti et al. 2009), where $\Omega$ is the solid angle of the spectral extraction regions, which is typically between 100 and $200 \operatorname{arcmin}^{2}$ beyond $27^{\prime}$. Thus, the measured residual CXB is in a very good agreement with the expected value.

We modeled the GXB as an absorbed two-thermalcomponent (APEC; Smith et al. 2001): one for the cool halo and the other for the hot halo. We fixed the metallicity at one solar, the redshift at zero, and the absorption to the Galactic value (Willingale et al. 2013). The two temperatures were each thawed and tied in all re-
63-82 arcmin

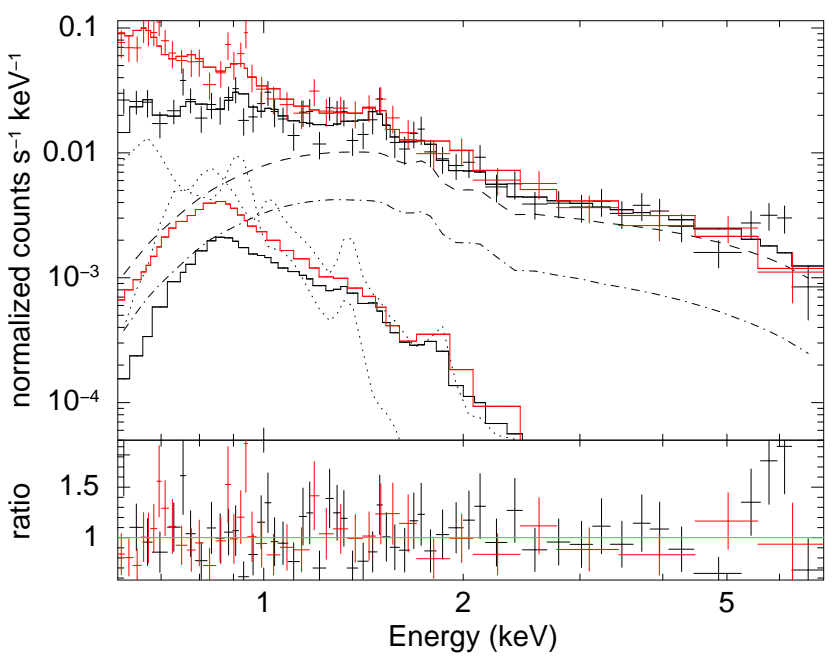

FIG. 2.- Representive Suzaku spectra covering the region around $R_{200}$. The FI and XIS1 data are shown in black and red, respectively. The solid lines following the data are the sum of all the model components. The two solid lines below the data indicate the ICM component. For clarity, we only show the GXB (the two dotted lines), the CXB (dashed), and the residual signal from the removed point sources (dash-dotted) components for the FI data. The error bars of the data are at $1 \sigma$.

gions. The best-fit temperatures are $T_{\mathrm{CH}} \approx 0.14 \mathrm{keV}$ and $T_{\mathrm{HH}} \approx 0.5 \mathrm{keV}$ for the cool halo and hot halo, respectively. The Antlia Cluster is located at a low Galactic latitude of $19^{\circ}$; such a high $T_{\mathrm{HH}}$ has been observed in other low Galactic latitude observations (Yoshino et al. 2009). We have checked the systematic uncertainty of the hot halo temperature by fixing $T_{\mathrm{HH}}$ at $0.3 \mathrm{keV}$, and this uncertainty was included in our analysis (Appendix B). The normalizations of the cool and hot halos were thawed and untied in all regions to take into account the angular variation. We do not include the very soft $\sim 0.08 \mathrm{keV}$ component for the Local Bubble, because it was not detected in our data analysis, possibly due to the $0.6 \mathrm{keV}$ cut-off energy we used. Sun et al. (2009) found a correlation between the GXB surface brightness and the ROSAT RASS R45 flux (in ROSAT PI channels 5290). The R45 flux in an annulus of $1^{\circ}-2^{\circ}$ centered at Antlia is $211 \times 10^{-6}$ counts s$^{-1}$ arcmin $^{-2}$, while in a circular region of a 0.2 radius centered at the EB field is $254 \times 10^{-6}$ counts s$^{-1}$ arcmin $^{-2}$. From spectral analysis, we measured the GXB surface brightness to be (1.1$1.2) \times 10^{-11} \mathrm{erg} \mathrm{s}^{-1} \mathrm{~cm}^{-2} \mathrm{deg}^{-2}$ in $0.47-1.21 \mathrm{keV}$ beyond $1^{\circ}$ from Antlia, which is in excellent agreement with the expected value shown in Figure 2 of Sun et al. (2009).

SWCX mainly produces line emission in the soft band $\lesssim 2 \mathrm{keV}$. We have filtered out soft X-ray flares potentially caused by SWCX, but the steady emission might also be contaminated by a constant level of SWCX. Thus, we include eight Gaussian models to take into account for the SWCX emission lines between $0.6-2.0 \mathrm{keV}$. The energies of the lines were fixed to those in Table 2 of Bautz et al. (2009). The Gaussian widths were fixed to zero. All the normalizations were thawed.

In the spectral analysis, we included both the ICM and background models for all regions, except for the local background field (EB) where only the background model was used. We simultaneously fitted the spectra from all 
the different regions in all the pointings (E0-EB). The unresolved CXB and SWCX are subject to cosmic variance and time variation, respectively. Therefore, we did not tie the CXB and SWCX normalizations for different regions, but only tied them for different detectors in the same spectral region. The ICM temperature, metallicity, and normalization were also not tied for different regions, but tied for the different detectors in the the same spectral region, with the metallicity beyond $18^{\prime}$ fixed to a constant value as mentioned above. Representive spectra covering the region around $R_{200}$ are shown in Figure 2

\section{SURFACE BRIGHTNESS PROFILE}

Soft X-ray emission can be strongly enhanced by SWCX or particle-induced background. Calibration uncertainty of the response files can also bias the emission measurement. To test whether there is significant systematic uncertainty in the soft X-ray emission measured with Suzaku, we have extracted the surface brightness profile from the ROSAT X-ray All-Sky Survey (RASS) using the same regions as the Suzaku analysis (Figure 3). We simulated the RASS surface brightness profile using the ROSAT response file and the best-fit Suzaku model. The simulated profile is consistent with the RASS data, suggesting that the soft X-ray emission measured with Suzaku is reliable.

The surface brightness profiles in the soft $(0.6-2 \mathrm{keV})$ and hard $(2-7 \mathrm{keV})$ energy bands are shown as black solid circles in Figure 4. NXB has been subtracted in these profiles. We also subtracted the local background (EB: the last data bin), and created the corresponding local background subtracted surface brightness profiles (red open circles), while the error of the local background has been added in quadrature. The local background subtracted surface brightness profile of the soft band (0.6$2 \mathrm{keV}$ ) decreases from the center to a minimum value at about $54^{\prime}$, and then flattens beyond that. The soft emission at the outermost two bins $\left(63^{\prime}-98^{\prime}\right)$ is each above the local background by about $7 \sigma$. Spectral analysis suggests that the flattening emission is at least partially caused by the slight enhancement of SWCX, and therefore the ICM contribution in the outer regions cannot be determined from this surface brightness profile. The local background subtracted surface brightness profile of the hard band $(2-7 \mathrm{keV})$ fluctuates around a small value beyond $\sim 20^{\prime}-30^{\prime}$, where the CXB dominates the emission.

In the left panel of Figure 5 the surface brightness profiles of different components in the soft band (0.6$2 \mathrm{keV}$ ) calculated using the spectral fitting models are shown. We also show the NXB component, which was subtracted from the data before the spectral fitting. For clarity, only the NXB of the XIS1 detector, which has a higher noise level than the XIS0 or XIS3 detectors, is plotted. For the ICM component, the solid line is the best-fit profile and the shaded regions correspond to its confidence regions calculated using the uncertainties of the APEC normalizations (Appendix A); both statistical and systematic errors are included. Both the GXB and CXB dominate the soft emission in the outer regions and are flat in the outer regions, while the residual signal from removed point sources (Res. Pt. in Figure 5) is typically the least dominant component everywhere. The NXB is almost always lower than the GXB and $\mathrm{CXB}$, and its

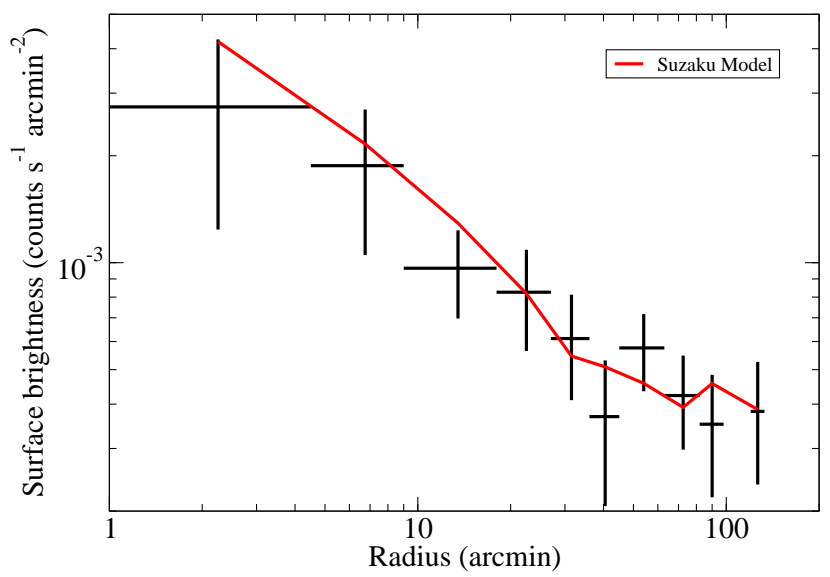

FIG. 3. - RASS surface brightness profile in the standard R47 band $(\sim 0.5-2 \mathrm{keV})$ extracted with the same Suzaku regions is shown in black data points. The red line is the simulated RASS profile using the best-fit Suzaku model and the ROSAT spectral response.

systematic uncertainty is $\lesssim 5 \%$. At radii beyond $\sim 54^{\prime}$, the surface brightness of the SWCX is comparable to the ICM. The SWCX is in fact enhanced beyond that radius. This is supported by the fact that the solar proton fluxes measured by $A C E$ are about a factor of two to eight higher during the observations for regions beyond $63^{\prime}$ compared to those between $9^{\prime}$ and $45^{\prime} 18$ Ignoring the SWCX component will overestimate the ICM emission in the outer regions.

The right panel of Figure 5 shows that the hard-band $(2-7 \mathrm{keV})$ surface brightness profiles. The NXB is the dominant component. The NXB subtracted profile ("Total" in the figure) is almost completely dominated by CXB beyond $\gtrsim 20^{\prime}-30^{\prime}$. Nevertheless, the very hard and unresolved $\mathrm{CXB}$ can be separated from the rest of the soft emission spectroscopically.

\section{TEMPERATURE PROFILE}

The projected and deprojected temperature profiles are shown in Figure 6, with temperature error bars including both statistical and systematic uncertainties (Appendix B). The projected temperature drops from the central region of $\sim 2$ to $\sim 0.7 \mathrm{keV}$ at the edge of the detection region of $\sim 100^{\prime}(1.2 \mathrm{Mpc})$. Such a declining temperature is typical in other clusters and groups. The nearly isothermal central region, the slowly decreasing outer temperature, and the smaller emission contribution from the outer regions ensure that the projected temperature profile is similar to the true deprojected temperature profile.

We fitted the global temperature between $\sim 0.2-1 R_{500}$ for pie regions with the same angular width, and the best-fit temperature is $1.54_{-0.13}^{+0.27} \mathrm{keV}$. This global temperature is in fact used to determine the radius scale used throughout the paper (Section 11).

The temperature profiles of most of the regular or less disturbed clusters appear to be self-similar beyond

18 No proton flux data were available for the observations $<9^{\prime}$ and between $45^{\prime}$ and $63^{\prime}$. 

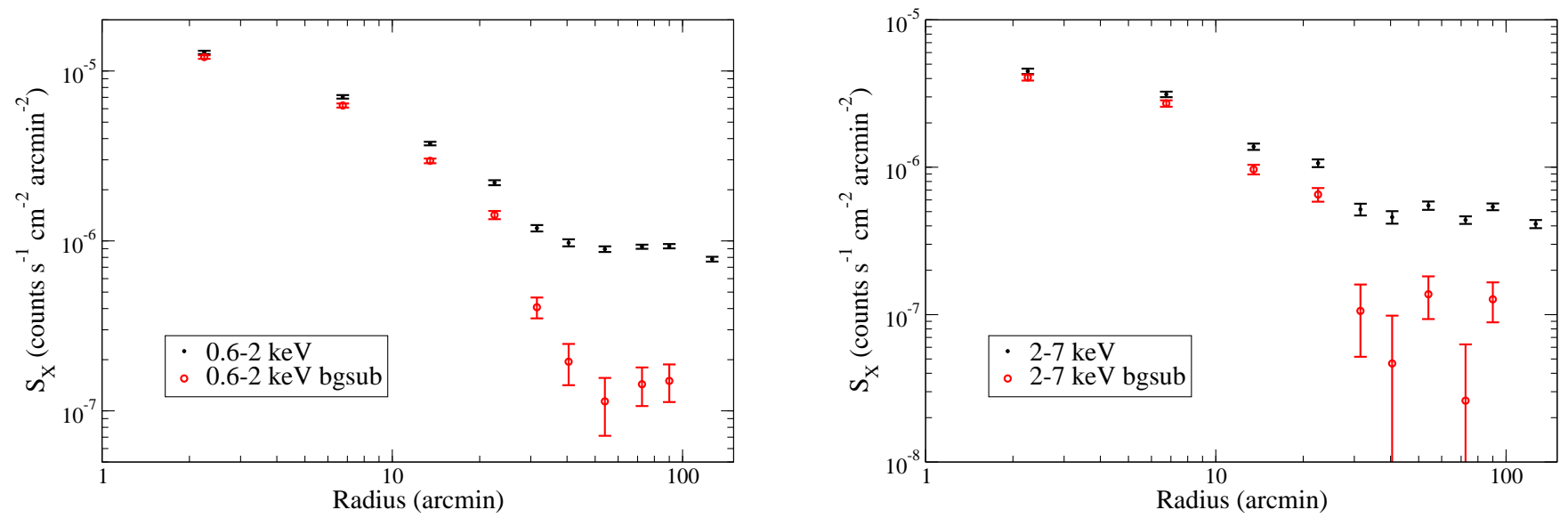

FIG. 4.- Left panel: surface brightness profiles of the unresolved diffuse emission in the $0.6-2 \mathrm{keV}$ band (black solid circles). Red open circles represent the profile with local background subtracted. Errors of the local background have been added in quadrature. Right panel: similar to the left panel but in the $2-7 \mathrm{keV}$ band.
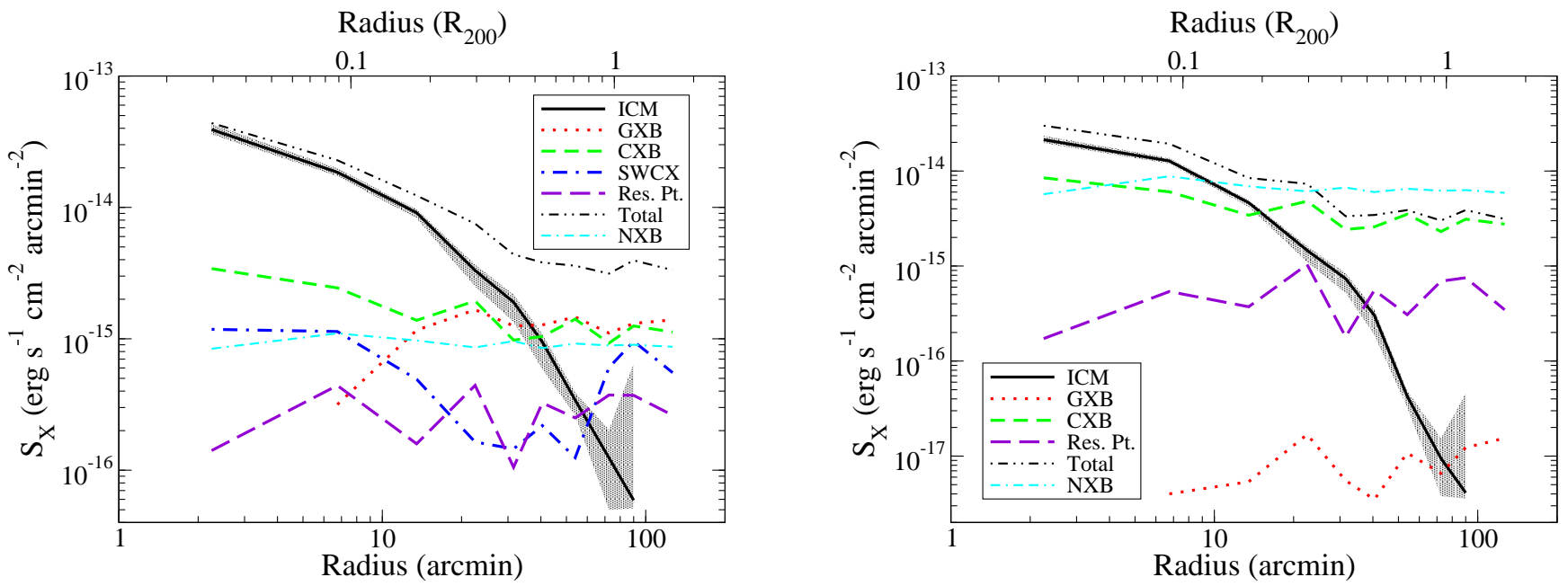

FIG. 5. - Left panel: surface brightness profiles of different components from 0.6-2 keV determined from spectral fitting. For the ICM component, the solid line is the best-fit profile and the shaded regions correspond to its confidence regions, including both statistical and systematic errors. The "Total" profile is the sum of all components except the NXB. For clarity, only the NXB of the XIS1 detector, which has a higher noise level than the XIS0 or XIS3 detectors, is plotted. Right panel: same as the left panel but from 2-7 keV.

$\geq 0.3 R_{200}$ (e.g., Reiprich et al. 2013). In Figure 6. we overplot the average scaled projected temperature profile measured with Suzaku and compiled by Reiprich et al. (2013) using a sample of 18 clusters with the following form:

$$
k_{\mathrm{B}} T /\left\langle k_{\mathrm{B}} T\right\rangle=1.19-0.84 R / R_{200},
$$

where we used the average Antlia temperature $\left\langle k_{\mathrm{B}} T\right\rangle=$ $1.54 \mathrm{keV}$ and $R_{200}=76^{\prime}$. The projected temperature profile of Antlia agrees very well with the average scaled temperature profile from the center out to $\sim R_{200}$. The projected temperature at about $1.2 R_{200} \approx 90^{\prime}$ is a factor of 2.3 higher than the average scaled profile, which can be explained by the local deviation in the temperature or the large scatter of temperature seen in the sample of Reiprich et al. (2013). For comparison, we also plot the projected temperature profile of Virgo measured by
Urban et al. (2011) using XMM-Newton, with its temperature scaled to match those of the Antlia Cluster. Other than the small-scale fluctuations caused by substructures, the general shape of the Virgo temperature profile agrees very well with that of the Antlia out to $\sim R_{200}$, as well as the average scaled profile. The agreement of these two low temperature groups with the sample of Reiprich et al. (2013), which includes mostly massive clusters $>4 \mathrm{keV}$, suggests that the temperature profile of low mass groups may also follow the self-similar profile of the more massive clusters beyond the core regions out to $R_{200}$. It should be noted that both Antlia and Virgo are measured in only one direction. More observations of galaxy groups will be needed to test the self-similarity near $R_{200}$.

\section{DENSITY, PRESSURE, AND ENTROPY PROFILES}




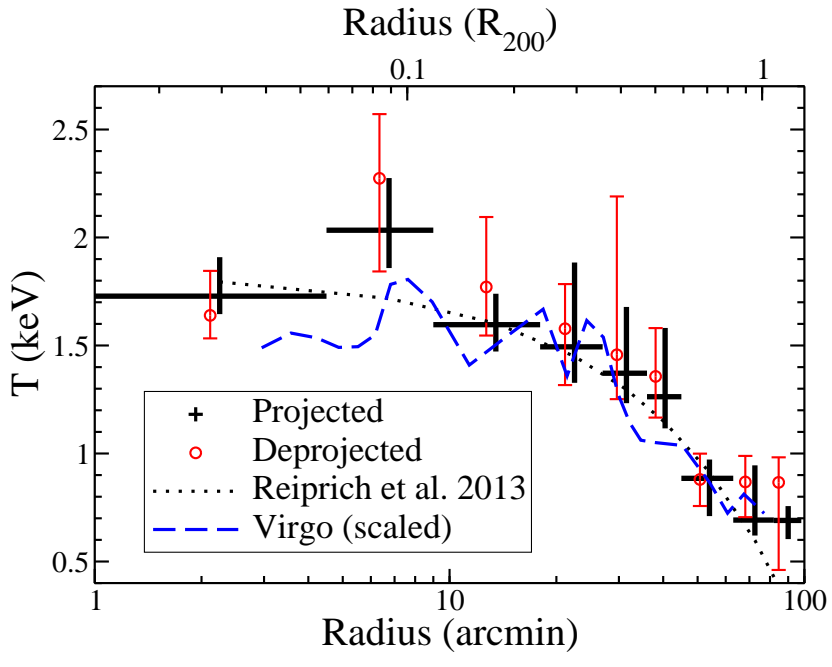

FIG. 6.- Projected (black crosses) and deprojected (red circles) temperature profiles of Antlia. Error bars for temperature include both statistical and systematic uncertainties. The deprojected temperature data points have been slightly shifted to the left for clarity. The dotted line is the average scaled profile of a sample of clusters measured with Suzaku (Reiprich et al. [2013). The blue dashed line is the Virgo profile with its projected temperature scaled by a factor of $\langle T$ (Antlia) $\rangle /\langle T$ (Virgo) $\rangle=1.54 / 2.3$ and radius in units of $R_{200}$ (Virgo) $=234^{\prime}$ (Urban et al. 2011). Note that the actual temperature of Virgo drops from its peak by a factor of three near the center $\left(\lesssim 10^{-3} R_{200}\right)$, which cannot be seen on the radial scale of this figure.

We used both the projected and deprojected spectral normalizations of the ICM component to constrain the electron density (Appendix A). The resulting electron density profile is shown in the upper panel of Figure 7

The density profile has a rather flat core within $\sim 10^{\prime}$ $20^{\prime}$ and steepens beyond that. The density decreases all the way beyond $R_{200}$ out to $\sim 100^{\prime}$. We fitted a power law to the density profile $\left(n_{e} \propto r^{-\alpha}\right)$ beyond $\sim 10^{\prime}$, and the power law index is $\alpha=1.75_{-0.24}^{+0.27}$. This is consistent with the density slope of $1.65-2.25$ at $R_{500}$ measured with 43 nearby galaxy groups using Chandra (Sun et al.|2009). It is not as steep as those of more massive galaxy clusters, which have a slope of $\sim 2$ at $R_{200}$ or even 3 at radii $\gtrsim R_{200}$ (e.g., Morandi et al. 2015). The density profile of Antlia in its outer regions is significantly steeper than that of Virgo $\left(\alpha_{\text {Virgo }}=1.21 \pm 0.2([19\right.$; Urban et al. 2011 $)$.

With the deprojected temperature and density profiles measured, we calculated the gas pressure profile, $P=n k T$, where $n$ is the total number density in the gas (both ions and electrons). We assume $n \approx 1.92 n_{e}$ for a fully ionized ICM. The pressure profile (middle panel in Figure 17) in the central $\sim 30^{\prime}$ is similar to the density profile because the temperature is quite uniform. Beyond that, the pressure profile is steeper due to the declining temperature.

Arnaud et al. (2010) found that more massive galaxy clusters with $M_{500} \gtrsim 10^{14} M_{\odot}\left(\right.$ or $T_{X} \gtrsim 2 \mathrm{keV}$ ) obey the universal pressure profile of the form:

$$
\frac{P(x)}{P_{500, \mathrm{ad}}}=\frac{P_{0}}{\left(c_{500} x\right)^{\gamma}\left[1+\left(c_{500} x\right)^{\alpha}\right]^{(\beta-\gamma) / \alpha}},
$$

19 The error of the Virgo density index has been converted to $90 \%$ confidence.

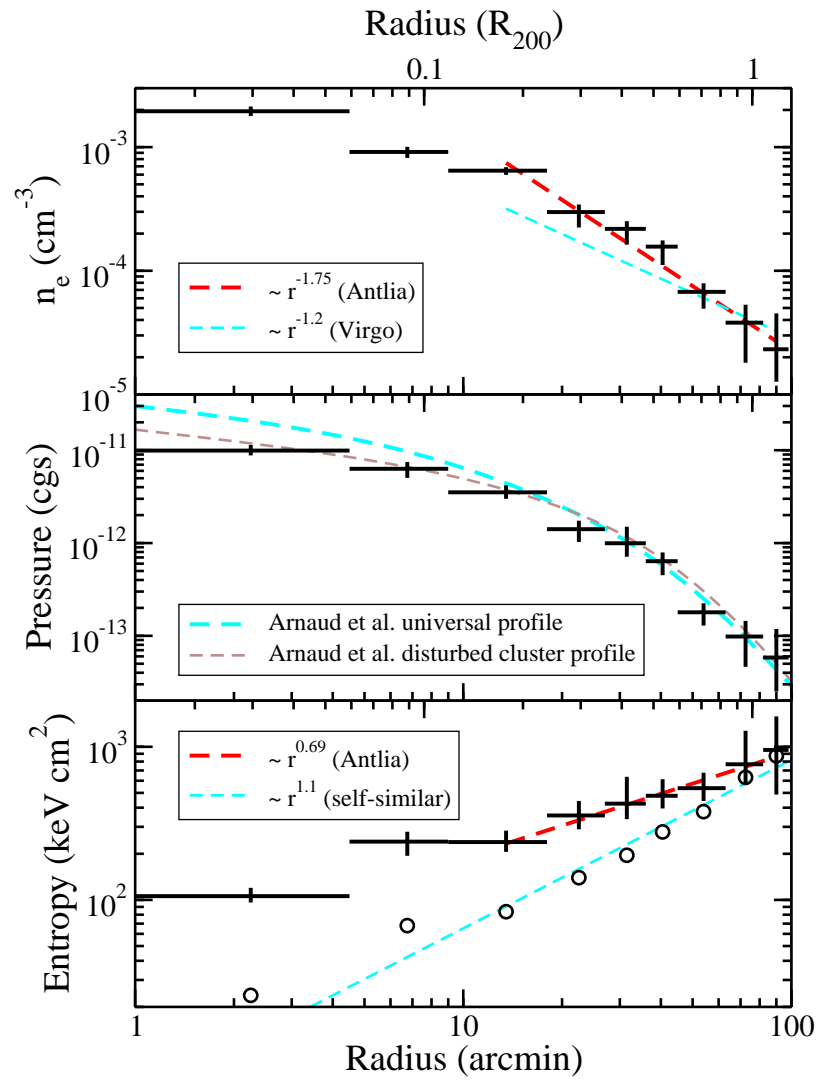

FIG. 7.- Upper panel: electron density profile of Antlia (black data), with a power law index of $\alpha=1.75_{-0.24}^{+0.27}$ beyond $\sim 10^{\prime}$ (thick red dashed). The thin cyan dashed line is the best-fit power law for Virgo $(\alpha=1.2 \pm 0.2)$ plotted in units of its $R_{200}$ (Urban et al. 2011). Middle panel: gas pressure profile of Antlia (black data). The standard universal pressure profile of clusters (thick cyan dashed) and the version with only morphologically disturbed clusters (thin brown dashed) are shown. Lower panel: entropy profile of Antlia (black crosses), with a power law index of $0.69_{-0.24}^{+0.22}$ beyond the core of $\sim 10^{\prime}$ (thick red dashed). The thin cyan dashed line is the gravity heating-only model with a power law index of 1.1. The circles are measured entropy multiplied by the gas correction factor of $\left.\left[f_{\text {gas }}(r) / 0.15\right)\right]^{2 / 3}$ (see text). Errors bars in density, pressure, and entropy include both statistical and systematic uncertainties.

with the characteristic pressure adjusted for the slight deviation from self-similar given by

$$
\begin{aligned}
P_{500, \text { ad }} & =1.65 \times 10^{-3} E(z)^{8 / 3} \\
\times & {\left[\frac{M_{500}}{3 \times 10^{14} h_{70}^{-1} M_{\odot}}\right]^{2 / 3+\alpha(x)} h_{70}^{2} \mathrm{keV} \mathrm{cm}^{-3}, }
\end{aligned}
$$

where $\alpha(x)=0.22 /\left[1+(2 x)^{3}\right]$ accounts for the breakdown of self-similarity, $x \equiv r / R_{500}$, and $E(z)=\sqrt{\Omega_{M}(1+z)^{3}+\Omega_{\Lambda}}$. Sun et al. (2011) found that the universal pressure profile can be extended to less massive groups. The thick cyan dashed line in the middle panel of Figure 7 shows the universal profile with the best-fitted parameters for all the cluster sample of Arnaud et al. (2010, i.e., $\left[P_{0}, c_{500}, \gamma, \alpha, \beta\right]=$ $\left.\left[8.403 h_{70}^{-3 / 2}, 1.177,0.3081,1.0510,5.4905\right]\right)$, with the radius scale of the Antlia Cluster $R_{500}=591 \mathrm{kpc}$. The pressure profile of Antlia agrees very well with the univer- 
sal profile beyond $\sim 30^{\prime}$, with a central deficit in pressure within that region. If we use the parameters for the morphologically disturbed clusters (i.e., $\left[P_{0}, c_{500}, \gamma, \alpha, \beta\right]=$ $\left[3.202 h_{70}^{-3 / 2}, 1.083,0.3798,1.4063,5.49\right] ; \quad$ thin brown dashed line; Arnaud et al. 2010), the pressure agrees over the full radial range. Urban et al. (2014) showed that in Perseus, where the directions are strongly disturbed by cold fronts or sloshing, the pressure profiles deviate significantly from the universal profile. The agreement in Antlia (in particular beyond $\sim 30^{\prime}$ ) suggests that the gas profile along the east direction may be a fair representation of the azimuthally averaged profile.

We also calculated the gas entropy parameter $K \equiv$ $k_{\mathrm{B}} T / n_{e}^{2 / 3}$, which reflects the thermodynamic history of the hot plasma (bottom panel in Figure 7). A self-similar model with only gravitational collapse heating predicts the entropy profile to be (Voit et al. 2005; Pratt et al. 2010):

$$
K=1.32 K_{200, \text { adi }}\left(\frac{r}{r_{200}}\right)^{1.1}
$$

where

$$
K_{200, \text { adi }}=362 \mathrm{keV} \mathrm{cm}^{2}\left(\frac{\bar{T}_{200}}{1 \mathrm{keV}}\right)\left(\frac{0.15}{f_{\mathrm{b}}}\right)^{2 / 3} E(z)^{-4 / 3},
$$

where $f_{\mathrm{b}}$ is the cosmic baryon fraction. The characteristic temperature here is related to the virial mass by $k_{\mathrm{B}} \bar{T}_{200} \equiv G M_{200} \mu m_{p} / 2 R_{200}$, where $\mu$ is the mean molecular weight per particle and $m_{p}$ is the proton mass. This self-similar model with the radius scale of the Antlia Cluster $R_{200}=887 \mathrm{kpc}$ is plotted as a thin cyan dashed line. The central entropy of cool core clusters can be biased low, while it can be biased high for non-cool core clusters. We therefore fitted a power law to the Antlia entropy profile excluding the $\sim 10^{\prime}$ core. The best-fit power law index is $0.69_{-0.24}^{+0.22}$, which is significantly flatter than that of $K \propto r^{1.1}$ for the self-similar model with only gravitational collapse heating. The entropy appears to be increasing from the center all the way out to $\sim R_{200}$. Going further, there is no evidence of entropy flattening or dropping beyond $R_{200}$, although the uncertainty is too large to be conclusive.

Within the $\sim 10^{\prime}$ core, the entropy is significantly higher than the self-similar model, which is typical for a non-cool core cluster. The overall profile is also clearly flatter than the self-similar model. Note that the magnitude of entropy beyond $R_{500}=51^{\prime}$ is in fact consistent with the gravity heating-only model. The flattening in Antlia should thus be caused by the increase of entropy inside that radius, which is different from other more massive clusters, where the flattening is caused by a lower entropy beyond $\sim R_{500}$ (e.g., Walker et al. 2013). The entropy of Antlia at $R_{500(2500)} 20$ is about $50(100) \%$ higher than the gravity-only self-similar model, which is similar to typical galaxy groups found by Sun et al. (2009). The extra entropy may be caused by any previous AGN feedback in the past, supernova feedback, preheating of gas before accretion, conduction that transfers heat from outer regions, low entropy gas cooling out of

$20 R_{2500} \sim 0.5 R_{500}$ for Antlia, as well as many nearby galaxy groups (Sun et al. 2009). the hot phase, or some combination of these processes (e.g., Sun et al. 2009; Pratt et al. 2010). At large radii beyond $\sim R_{500}$, there is no evidence of the entropy changing due to non-gravitational processes for Antlia. Note that due to the large temperature uncertainty, if the temperature beyond $R_{200}$ is biased high by a factor of two, the entropy will be reduced by the same factor, and the entropy profile can be flat beyond that.

Pratt et al. (2010) found that the general existence of a central entropy excess in clusters is connected to the gas faction. By introducing a gas correction factor, the corrected entropy matches the theoretical prediction better. In the lower panel of Figure 7 , the circles represent the measured entropy multiplied by a factor of $\left[f_{\text {gas }}(r) / 0.15\right]^{2 / 3}$, where $f_{\text {gas }}(r)$ is the gas fraction measured in Section 6 below. The corrected entropy profile of Antlia becomes largely consistent with the theoretical prediction.

\section{GAS AND HYDROSTATIC MASS PROFILES}

With the density profile measured, we can calculate the enclosed gas-mass profile by

$$
M_{\text {gas }}(<r)=4 \pi \int_{0}^{r} d r^{\prime} r^{\prime 2} \rho_{\text {gas }}\left(r^{\prime}\right)
$$

where $\rho_{\text {gas }}=\mu_{e} m_{p} n_{e}$ is the gas mass density, and $\mu_{e}$ is the mean molecular weight per electron determined from the gas abundances. We also calculated the HSE mass given by (e.g., Sarazin 1986)

$$
M_{\mathrm{HSE}}(<r)=-\frac{k T r}{\mu m_{p} G}\left(\frac{d \ln \rho_{\mathrm{gas}}}{d \ln r}+\frac{d \ln T}{d \ln r}\right) .
$$

For regions inside $\sim 20^{\prime}$, we directly applied equation (7) on the measured density and temperature to calculate $M_{\mathrm{HSE}}$. However, for regions beyond that, the uncertainty is very large due to the large uncertainties in the data. To capture the global behavior, we fitted power law models to the temperature and density profiles beyond $\sim 20^{\prime}$, $T \propto r^{\Gamma_{T}}$ and $\rho \propto r^{\Gamma_{\rho}}$; therefore equation (7) becomes

$$
M_{\mathrm{HSE}}(<r)=-\frac{k T r}{\mu m_{p} G}\left(\Gamma_{T}+\Gamma_{\rho}\right) \text {. }
$$

In calculating $M_{\text {gas }}$ and $M_{\mathrm{HSE}}$, we used the $10^{6}$ simulated density and temperature profiles, and assessed the median and errors in a Monte Carlo sense described in Appendix A. The mass profiles are shown in Figure 8.

The gas-mass increases from $3.4 \times 10^{10} M_{\odot}$ at $4^{\prime} .5$ to $4.6(7.5) \times 10^{12} M_{\odot}$ at $R_{500(200)}=51^{\prime}\left(76^{\prime}\right)$. Compared with the gas mass, the HSE mass increases with a flatter slope, with $M_{\mathrm{HSE}}\left(R_{500}\right)=5.5 \times 10^{13} M_{\odot}$ and $M_{\mathrm{HSE}}\left(R_{200}\right)=6.5 \times 10^{13} M_{\odot}$. If we use our adopted scale radius to calculate the scale mass $M_{\Delta} \equiv 4 \pi \Delta \rho_{c} R_{\Delta}^{3} / 3$, we find that $M_{500(200)}=5.9(7.9) \times 10^{13} M_{\odot}$, which is 7 (20)\% higher than $M_{\mathrm{HSE}}$ at $R_{500(200)}$. While the precise HSE mass bias from the total mass is uncertain, it can be biased low by $10-40 \%$ beyond $R_{500}$ (e.g., Mahdavi et al. 2013; Okabe et al. 2014). Because we only have measurements in one direction, azimuthal variation may also bias these mass estimations on the same order. Since we found that the HSE masses $M_{\mathrm{HSE}}$ are $7 \%$ and $20 \%$ lower than the scaled masses $M_{\Delta}$ calculated using our adopted scaled radii at $R_{500}$ and $R_{200}$, respectively, the deviations 


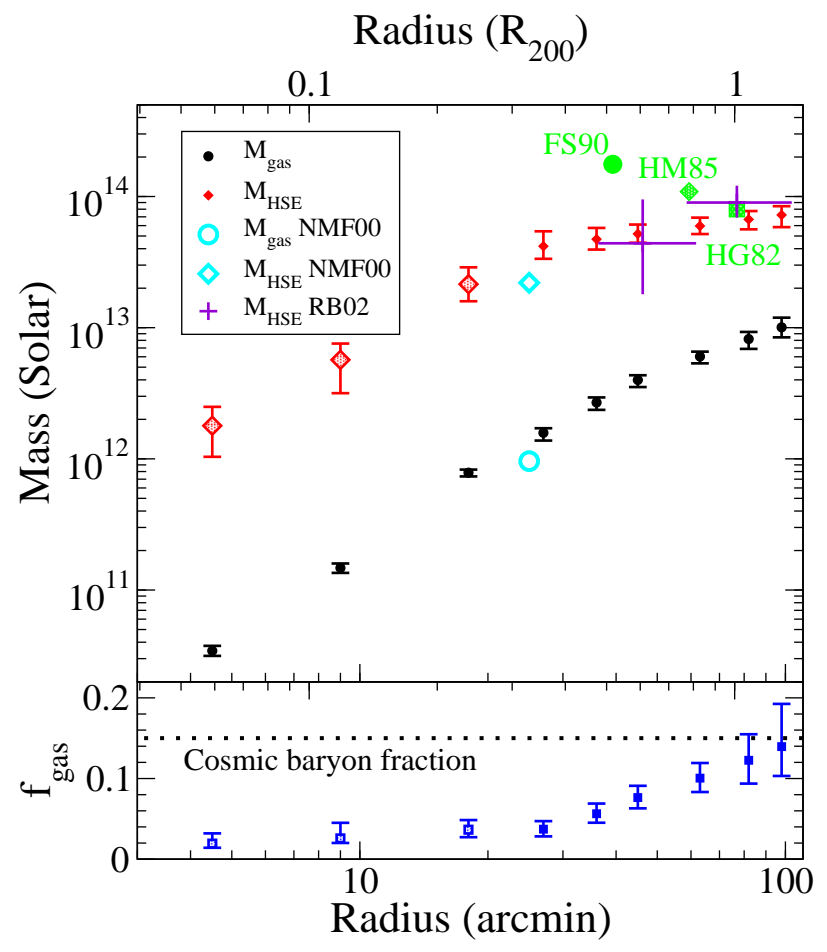

FIG. 8.- Upper panel: gas-mass $M_{\text {gas }}$ (small solid black circle) and HSE mass $M_{\mathrm{HSE}}$ (large red diamond using equation (7); small solid red diamond using equation (80) profiles of Antlia. X-ray measurements by Nakazawa et al. (2000, NMF00) are shown with large open cyan circles and diamonds. $M_{\mathrm{HSE}}$ estimated by Reiprich \& Böhringer (2002, RB02) is shown with violet crosses, with error bars scaled to $90 \%$ confidence. Dynamical mass estimations measured by Huchra \& Geller (1982, HG82), Hopp \& Materne (1985, HM85), and Ferguson \& Sandage (1990, FS90) are shown as shaded green points. All of the measurements have been rescaled to the Hubble constant or distance we adopted. Lower panel: gas fraction $\left[f_{\text {gas }}(<r) \equiv M_{\text {gas }} / M_{\mathrm{HSE}}\right]$ of Antlia. The horizontal dashed line shows the comic baryon fraction of 0.15 . Note that, as usual throughout the paper, the scaled radius $R_{200}$ on the top-axis is defined using the scaling relation (Section 1) instead of using the HSE mass determined in this section.

are insignificant compared to the mass biases introduced by azimuthal variation and HSE bias 21 Thus, we can adopt the scaled radii using the X-ray scaling relation.

In Figure 8, we also plot other mass measurements in the literature. All of the measurements have been rescaled with the Hubble constant or distance we used. Compared to previous X-ray measurements with ROSAT and ASCA (Nakazawa et al. 2000) at $\sim 0.5 R_{500}$, our $M_{\text {gas }}$ and $M_{\mathrm{HSE}}$ are about $40 \%$ and $60 \%$ higher, respectively. The difference in $M_{\text {gas }}$ might be partially due to their high assumed metallicity of $0.35 Z_{\odot}$, while our measured metallicity decreases from $\sim 0.4 Z_{\odot}$ down to $\lesssim 0.15 Z_{\odot}$ at $\sim 0.5 R_{500}$. A bias high in metallicity results in a lower normalization in the spectral fitting, and hence a bias low in gas density or gas mass (Appendix [B]). Another possible reason is due to their isothermal assumption when determining both $M_{\text {gas }}$ and $M_{\mathrm{HSE}}$, as well as the prior assumption of a dark matter poten-

21 Note that the scaled masses $M_{\Delta}$ are adopted using the scaling relation of Sun et al. (2009), while this scaling relation is also measured using the HSE method and is thus subjected to HSE bias. tial in their work. Reiprich \& Böhringer (2002) estimated the $M_{\mathrm{HSE}}$ at $R_{500}$, which is very consistent with our measurement. Their $M_{\mathrm{HSE}}$ at $R_{200}$ is about $40 \%$ higher. This could be due to the isothermal temperature of $1.18 \mathrm{keV}$ they assumed, which is biased high by a factor of about $1.2-2$ between $R_{500}$ and $R_{200}$, and hence $M_{\mathrm{HSE}}$ could be biased high. Mass estimations by assuming the optical galaxies in dynamical equilibrium at different characteristic radii are generally larger than our measured $M_{\mathrm{HSE}}$ (Huchra \& Geller 1982; Hopp \& Materne 1985; Ferguson \& Sandage 1990). However, the numbers of galaxies used in these measurements are very small $(\leq 21)$, and therefore the statistical uncertainties are quite large.

The stellar mass can be estimated from the total Kband luminosity. Lin et al. (2004) found that the K-band luminosity of all the galaxies inside $R_{200}$ is $L_{K, 200}=$ $1.54 \times 10^{12} h_{70}^{-2} L_{\odot}$ With a typical mass-to-light ratio of $M / L_{K}=0.95$ (Bell et al. 2003), the total stellar mass of the Antlia Cluster is therefore $M_{\star}=1.5 \times 10^{12} h_{70}^{-2} M_{\odot}$. Thus, the gas starts to dominate over the stellar component for radii $\gtrsim 26^{\prime} \approx 0.34 R_{200}$. The stellar mass is about $30(20) \%$ of the gas mass at $R_{500(200)}$.

The gas-mass fraction is defined as $f_{\text {gas }} \equiv M_{\text {gas }} / M_{\mathrm{HSE}}$ and is shown in the lower panel of Figure 8 . The gas fraction increases from about 0.02 near the center to about 0.14 at $\sim 100^{\prime}$. The gas fraction does not exceed the cosmic baryon fraction 23 even slightly beyond $R_{200}$, suggesting that gas clumping or fluctuations are not significant in this direction of the Antlia Cluster. Note that the HSE mass can be biased low by $>30 \%$ beyond $R_{500}$, and therefore the true gas fraction is likely to be even lower at the outer boundary (Okabe et al. 2014). The baryon fraction, which includes both the gas and stellar components, is at most a factor of 1.2 of the gas fraction at $R_{200}$, and thus will not alter our conclusion.

\section{ELECTRON-ION EQUIPARTITION AND COLLISIONAL IONIZATION TIMESCALES}

Because of the low density in the outskirts of galaxy clusters/groups, the collisional timescales can be longer than the dynamical timescales. Thus, after hot gas has passed through an accretion shock, electrons and ions may not be in equipartition (e.g., Fox \& Loeb 1997; Wong \& Sarazin 2009) and collisional ionization equilibrium (CIE) may not be reached (Wong et al. 2011). The electron-ion equipartition timescale is estimated to be (Spitzer 1962, p. 135)

$t_{e i} \approx 7.0 \times 10^{8} \operatorname{year}\left(\frac{T_{e}}{10^{7} \mathrm{~K}}\right)^{3 / 2}\left(\frac{n_{e}}{10^{-5} \mathrm{~cm}^{-3}}\right)^{-1}\left(\frac{\ln \Lambda}{40}\right)^{-1}$,

where $\ln \Lambda$ is the Coulomb logarithm. The collisional ionization timescale for most elements of astrophysical interest is estimated to be (Smith \& Hughes 2010)

$$
t_{\mathrm{CIE}} \approx 3 \times 10^{9} \text { year }\left(\frac{n_{e}}{10^{-5} \mathrm{~cm}^{-3}}\right)^{-1} .
$$

These two timescales of Antlia are plotted in Figure 9 We compare these to the shock-elapsed timescale (i.e.,

22 We rescaled the luminosity according to the distance we used.

23 We adopted the cosmic baryon fraction of 0.15 measured by Planck (Planck Collaboration 2014). 


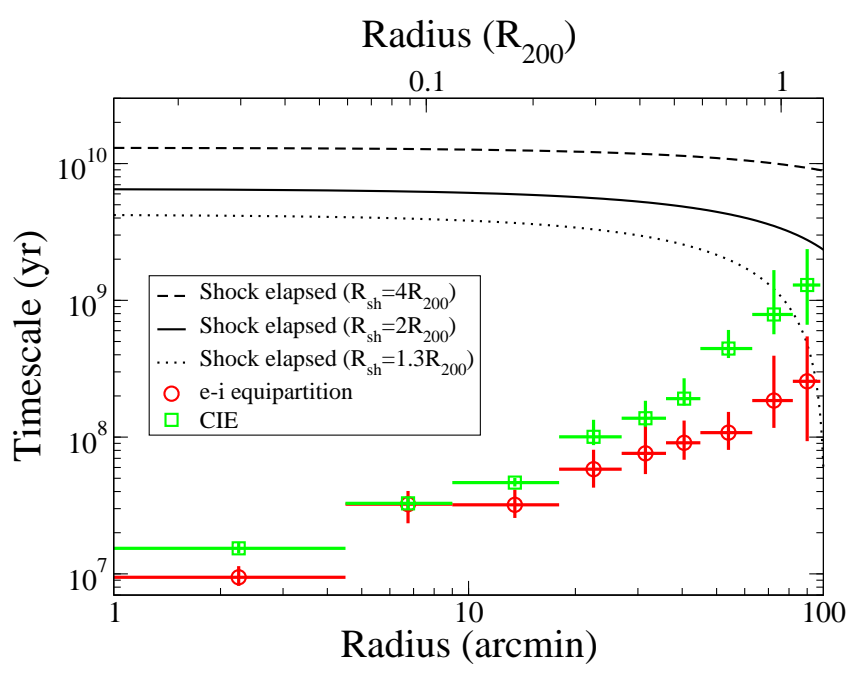

Fig. 9.- Electron-ion equipartition (red circles) and collisional ionization (green squares) timescales for Antlia. The shock-elapsed timescales, assuming the shock radius is at $4 R_{200}, 2 R_{200}$, and $1.3 R_{200}$ are shown in black dashed, solid, and dotted lines, respectively. We expect ions and electrons to share the same temperature or ionization in CIE, where the equipartition or the collisional ionization timescale is shorter than the shock-elapsed timescale, respectively.

the timescale since the gas has passed the accretion shock)

$$
t_{\mathrm{sh}}(r)=\frac{r-R_{\mathrm{sh}}}{v_{\mathrm{sh}}},
$$

where the shock velocity $v_{\mathrm{sh}} \approx v_{\text {infall }} / 3$. The infalling velocity can be estimated as $v_{\text {infall }} \approx \sqrt{2 k_{\mathrm{B}} T / \mu m_{p}}$ (e.g., Takizawa 1998). We take the maximum temperature of Antlia to estimate the minimum $t_{\mathrm{sh}}$. From numerical simulations, typical accretion shock radii are between $1.3 R_{200}<R_{\text {sh }}<4 R_{200}$. We adopted a shock radius of $R_{\mathrm{sh}}=2 R_{200}$ to be consistent with the simulations of Wong \& Sarazin (2009). We also consider the upper and lower limits of $R_{\mathrm{sh}}=4 R_{200}$ and $1.3 R_{200}$, respectively.

Figure 9 shows that the electron-ion equipartition time $t_{e i}$ is always shorter than the shock elapsed time $t_{\mathrm{sh}}$ at radius $\lesssim 1.2 R_{200} \approx 90^{\prime}$. This suggests that electrons and ions should have enough time to share energy, and hence they should have the same temperature $\left(T_{e}=T_{i}\right)$ inside that radius. This is consistent with the simulations of Wong \& Sarazin (2009) that the non-equipartition effect should be small at radii $\lesssim R_{200}$, but only becomes important beyond that. However, if the accretion shock radius is very small $\left(R_{\mathrm{sh}}<1.3 R_{200}\right)$ or internal accretion shocks develop close to $R_{200}$, a non-equipartition effect can be important near $R_{200}$.

The collisional ionization time $t_{\mathrm{CIE}}$ is about four to five times longer than $t_{e i}$ near $R_{200}$. For $R_{\mathrm{sh}} \lesssim 1.3 R_{200}$, $t_{\mathrm{CIE}}$ is longer than $t_{\mathrm{sh}}$ at radii $\gtrsim R_{200} \approx 76^{\prime}$. A nonequilibrium ionization (NEI) state is possible around $R_{200}$, and might be detected with high-resolution X-ray spectrometer (see., e.g., Wong et al. 2011). However, if the accretion shock radius is larger $\left(R_{\mathrm{sh}} \gtrsim 1.3 R_{200}\right), t_{\mathrm{CIE}}$ is shorter than $t_{\mathrm{sh}}$, and therefore the gas is likely to be in CIE for radii $\lesssim R_{200}$.

\section{8. $f_{\text {gas }, 200}-T_{500}$ AND $K_{200}-T_{500}$ RELATIONS}

Measuring the gas fraction of galaxy clusters and groups is of great interest. It depends on the fraction that has converted to stars and the gas expelled by heating or during the cluster or group formation; thus it can be used to test structure formation theories. The gas fraction has also been used to constrain cosmological parameters (e.g., Allen et al. 2008). X-ray studies of large samples of individual clusters and groups have been used to constrain the $f_{\text {gas }}-T$ relations out to $R_{500}$ (see, e.g., Sun 2012). Another important quantity is entropy, which reflects the thermodynamic history of hot gas. When measuring the entropy at small radii $\lesssim 0.15 R_{500}$, the $K_{\Delta}-T_{500}$ relations of groups and clusters have been found to deviate from the gravity heating-only baseline model, with a large scatter for low mass groups. The scaling relations become consistent with the baseline model as the radii approach $R_{500}$ (Sun et al. 2009; Pratt et al. 2010). We extend earlier work to examine the $f_{\text {gas, } 200}-T_{500}$ and $K_{200}-T_{500}$ relations with the gas fraction and entropy measured at $R_{200}$.

We complied the enclosed gas-mass fractions $\left(f_{\text {gas }, 200}\right)$ and entropies $\left(K_{200}\right)$ of galaxy groups and clusters measured out to $R_{200}$ using Suzaku data published in the literature. One of the clusters, Virgo, which was measured with XMM-Newton, is also included to increase the number of data points in the sparse low mass group range. The compiled results are listed in Table 2 The scaled temperatures $T_{500}$ were taken from the work listed in column 3 of Table 2. The $T_{500}$ values reported are average temperatures measured from $0.15 R_{500}$ to $R_{500}$ or close to this range. The scaled radii $R_{200}, f_{\text {gas,200 }}, K_{200}$, and redshift $z$ were taken from the work listed in column 8 of Table 2 When there is no value at $R_{200}$ reported explicitly, we evaluated $f_{\text {gas,200 }}$ and $K_{200}$ at $R_{200}$ by interpolation or extrapolation in log space using the two nearest data points in the radial profile. Extrapolations were done for two clusters with $R_{200}$ no more than $\sim 10 \%$ beyond the outer boundary of the data. The extrapolated values are enclosed in parentheses in Table 2.

It would be ideal if the observations were taken in relaxed directions or azimuthally averaged over all directions to minimize biases along merging or filament directions. However, most of the observations only covered one or a few narrow directions due to the expensive exposure required. Therefore, to increase the sample size, we also included clusters with observed directions along merging or filament directions. The data of the merging or filament directions used in our sample do not appear to introduce significant biases to the scaling relations (see Figures 10 and11below). When there was more than one direction reported in the literature, we chose the relaxed direction if there was a significant difference between the relaxed and non-relaxed directions. Otherwise, the azimuthal average quantities were used. The last column of Table 2 describes the directions of the observations. The errors of all data have been converted to $90 \%$ confidence range.

Figure 10 shows the $f_{\text {gas }, 200}-T_{500}$ relations for a wide range of temperatures. We fitted the data with the Bivariate Correlated Errors and Intrinsic Scatter (BCES) method (Akritas \& Bershadv 1996). The $(Y \mid X)$ regression was used because the temperature errors are generally smaller than the gas fraction errors. If we consider only those with total mass measured using the HSE 


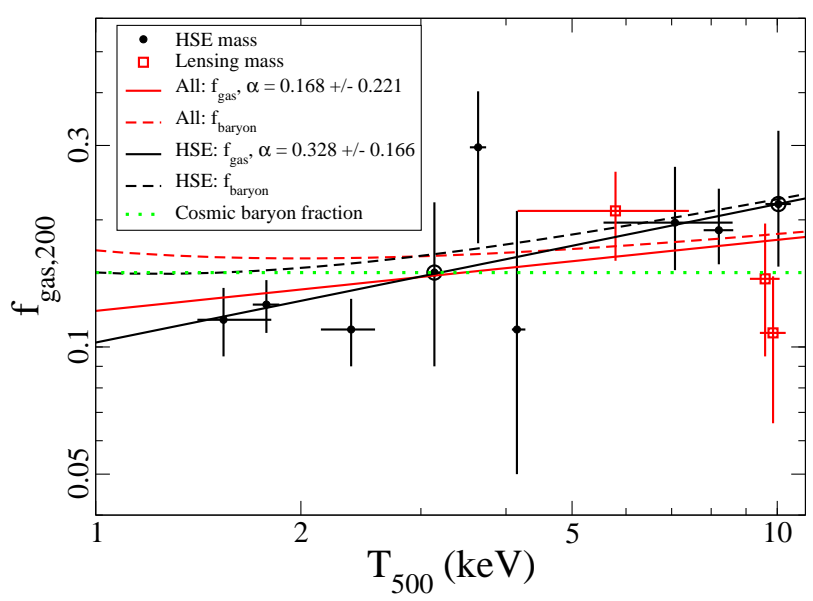

Fig. 10.- Enclosed gas fraction within $R_{200}$ vs. $T_{500}$. Black open circles indicate clusters measured along merging or filament directions. The black solid line is the best fit to groups and clusters with masses measured using the HSE method. The red solid line is the best fit to all groups and clusters in the sample (masses measured with either HSE or weak lensing methods). The corresponding dashed lines are the total baryon fraction within $R_{200}$, calculated by adding the gas fraction with the stellar mass fraction. Note that the small sample size of weak lensing mass measurements can introduce a strong bias.

method, the best-fit power law slope is $0.328 \pm 0.166$, suggesting an increasing $f_{\text {gas }, 200}$ as temperature increases. At $R_{500}$, Sun et al. (2009) found that the slope is $\sim 0.16$ 0.22 , while it can be as steep as 0.32 , as found by Lovisari et al. (2015). The error of the slope at $R_{200}$ is too large to tell if there is any difference from those at smaller radii. If we include the three clusters with total masses measured using the weak lensing method, the best-fit power law slope decreases to $0.168 \pm 0.221$, and hence the correlation becomes insignificant. Note that adding these three lensing clusters can introduce biases to the results, because the values of $f_{\text {gas }, 200}$ for other clusters might decrease with weak lensing mass measurements. For example, if the ratio of HSE mass and weak lensing mass are the same for all groups or clusters, the $f_{\text {gas,200 }}$ slope of the HSE sample will be the same as that of the weak lensing sample. Note also that only two reliable weak lensing mass measurements are available in these data, and therefore our discussions related to weak lensing measurements can be strongly biased.

We estimated the enclosed baryon fraction $f_{\text {baryon }}$ by adding the enclosed stellar fraction at $R_{200}$ measured by Andreon (2010). The results are shown as dashed lines in Figure 10. For the sample with only HSE mass measurements, $f_{\text {baryon }}$ rises from 0.15 for $1 \mathrm{keV}$ groups to 0.22 for $10 \mathrm{keV}$ clusters, but note that the uncertainly is too large to confirm the trend or to tell whether there is an apparent baryon excess for the massive clusters. The excess in $f_{\text {baryon }}$ for massive clusters with HSE mass measurements is only marginally significant. There is no strong evidence of significant clumping around $R_{200}$. When including the clusters with lensing mass measurements, $f_{\text {baryon }}$ is essentially flat. The baryon fractions of massive clusters are consistent with the cosmic value within $\sim 20 \%$, which is smaller than the uncertainty of

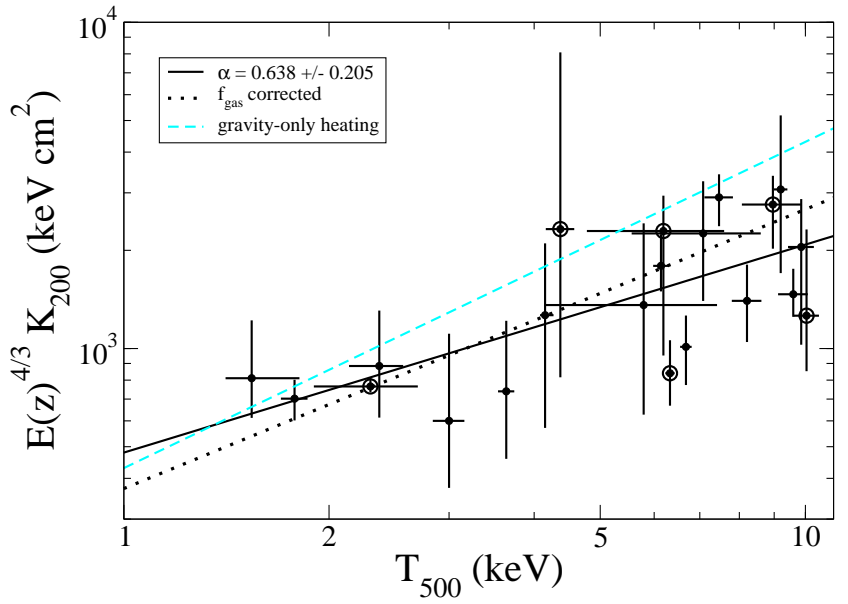

FIG. 11. - Entropy at $R_{200}$ vs. $T_{500}$. Black open circles indicate clusters measured along merging or filament directions. The black solid line is the best fit to all groups and clusters in the sample. The black dotted line is the gas corrected relation. The cyan dashed line is the baseline model.

the measurement. Therefore, there is no evidence of missing baryons in all mass ranges.

The $K_{200}-T_{500}$ relation is plotted in Figure [11. We have also plotted the baseline entropy model using equations (4) and (5), where we assume $R_{200} / R_{500}=1.5$ when converting $\bar{T}_{200}$ to $\bar{T}_{500}$. Using the BCES method, the best-fit power law slope is $0.638 \pm 0.205$, which is significantly smaller than the baseline entropy model of 1 . The slope is also smaller than that of the observed $K_{500^{-}}$ $T_{500}$ relation, which was found to be $0.994 \pm 0.054(1 \sigma)$ by Sun et al. (2009) and $0.92 \pm 0.24(1 \sigma)$ by Pratt et al. (2010). For low temperature groups with $T_{500} \lesssim 2.5 \mathrm{keV}$, $K_{200}$ is consistent with the baseline model. Above that, $K_{200}$ is significantly smaller. While there are significant entropy excesses for groups at $R_{500}$ compared to the baseline model (Sun et al. 2009), we do not see any excess for groups at $R_{200}$. This suggests that non-gravitational heating is important at radii $\lesssim R_{500}$ but not around $R_{200}$. There is also some evidence of entropy excess in more massive clusters at $R_{500}$ shown by Sun et al. (2009). However, Figure 11] shows that the entropy at $R_{200}$ is clearly smaller than the baseline model. Such an entropy deficit in massive clusters measured with Suzaku was discussed by various authors (see Section [9.2), although Eckert et al. (2013) did not find a significant deficit in their stacked ROSAT and Planck sample. Okabe et al. (2014) found that the entropy scaling relation has a slope that agrees with the baseline model near $\sim R_{200}$, which is steeper than our finding, although they also noted that the normalization is lower than the baseline model. If we only take the three clusters (Hydra A, A1835, A1689) that were also used in their four clusters sample, the $K-T$ slope is closer to 1 . Thus, the slope found in Okabe et al. (2014) might be overestimated due to their small sample size.

We corrected the best-fit entropy by introducing the same gas correction factor as in Section 5. We used $f_{\text {gas } 200}$ from the HSE sample (black solid line in Figure [10) so that a larger correction can be made. The 
TABLE 2

Clusters with measurements out to $R_{200}$ with Suzaku, Plus Virgo (measured with XMM-Newton).

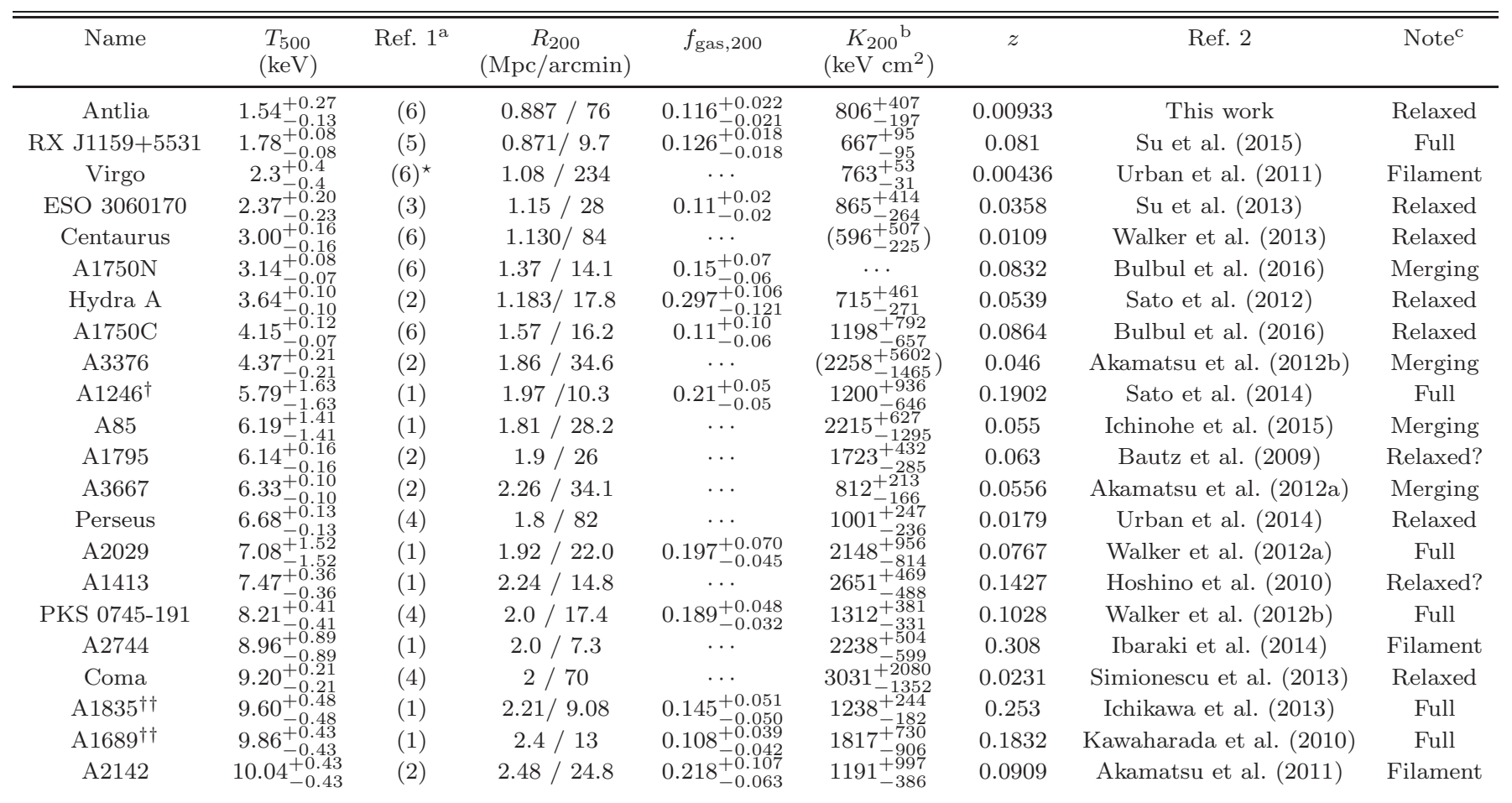

${ }^{a}$ Reference of $T_{500}$ taken from: (1) Morandi et al. (2015), (2) Vikhlinin et al. (2009), (3) Sun et al. (2009), (4) De Grandi \& Molendi (2002), (5) Humphrev et al. (2012), (6) same as column (8).

$\mathrm{b}$ The values in parentheses are from extrapolation (see text).

c Directions of the observations indicated by the authors in column (8). "Relaxed" includes directions away from the merging or filament axis. "Merging" includes either direction along the merging axis. "Full" indicates average value using four or more directions. The "?" symbol indicates that the condition is not explicitly written by the authors, but it is inferred by the content of the paper.

* Error of $T_{500}$ was not reported, and we estimated it from the fluctuations in its temperature profile.

$\dagger f_{\text {gas }}$ is measured using the weak lensing mass instead of HSE mass, where the weak lensing mass is estimated using a weak lensing template profile from a sample of low mass clusters rather than measured directly.

$\dagger^{\dagger} f_{\text {gas }}$ of the cluster/group calculated using the weak lensing mass instead of HSE mass.

gas-corrected entropy is shown as a dotted line of Figure 11. The corrected entropy has a slope of $0.86 \pm 0.23$, but the uncertainty is too large to tell whether it deviates from 1 of the baseline model. The corrected entropy for massive clusters is still lower than the baseline model, suggesting that the entropy deficit at $R_{200}$ is not fully correlated to the gas fraction, unlike those connections found at smaller radii (Pratt et al. 2010). Thus, the entropy deficits for massive clusters might require biases or deviations in temperature measurements.

\section{DISCUSSION}

\subsection{Diversity of Groups Out to $R_{200}$}

The Virgo Cluster (or group) is a cool core cluster with a similar temperature (2.3 keV; Urban et al. 2011) and mass $\left(M_{500} \approx 10^{14} M_{\odot}\right.$ : Walker et al. 2013) as Antlia. Virgo is the nearest cluster and Antlia is the nearest noncool core cluster, making them an ideal pair to compare the difference between the cool core and non-cool core groups out to the virial regions with the best spatial resolution. Note that the thermodynamic properties of Virgo were also studied in one direction (north) out to $\sim R_{200}$ by Urban et al. (2011). Virgo was studied along its major axis in X-rays where gas could be accreted faster, while Antlia was studied between the major and minor axes.

Besides the fluctuations of temperature due to small-scale structures resolved with XMM-Newton (Urban et al. 2011), we found that both Antlia and Virgo follow the same average scaled temperature profile of many clusters compiled by Reiprich et al. (2013) between $\sim 0.1$ and $1 R_{200}$. The pressure profile of Virgo shows significant fluctuations, but the general trend appears to be flatter and slightly higher than the universal pressure profile beyond $\sim 0.4 R_{200}$ (Figure 6 in Urban et al. 2011), in contrast to Antlia where the universal pressure profile is closely followed. Thus, the density slope is flatter than that of Antlia as $n \propto P / T$, which can be seen in Figure 7. The entropy profile of Virgo is also flatter as $K \propto T / n^{2 / 3}$. Figure 10 in Walker et al. (2013) shows that, in spite of the large fluctuations, the entropy of Virgo is almost constant beyond $\sim 0.3 R_{200}$. Similar to Antlia, the entropy beyond $\sim R_{500}$ approaches the gravity heating-only model. There appears to be some weak evidence that the entropy might be dropping slightly around $R_{200}$, but the fluctuations are too large to be conclusive. Note that the last data point in the density in Figure 6 of Urban et al. (2011) is likely biased high by about a factor of two due to the ringing effect in deprojection (see text in their paper), and thus the entropy in 
Figures 10 and 12 of Walker et al. (2013) is biased low by about a factor of $2^{2 / 3}=1.6$. Taking this into account eliminates the entropy deficit at $R_{200}$ in Virgo, as well as the claim of clumping based on entropy deficit or self-similar considerations (Walker et al. 2013). Thus, non-gravitational effects in the Virgo outskirts are probably not very strong, similar to Antlia. The flatter and stronger entropy excess inside $\sim R_{500}$ in Virgo suggests a stronger heating compared to Antlia, perhaps due to the strong AGN feedback from the supermassive black hole in M87.

We also compared the dynamically young Antlia to the dynamically evolved fossil groups that ESO 3060170 studied along one direction ( $\mathrm{Su}$ et al. 2013) and RX $\mathrm{J} 1159+5531$ studied in all directions (Humphrey et al. 2012; Su et al. 2015). RX J1159+5531 (M $M_{500} \sim 6 \times$ $\left.10^{13} M_{\odot}, z=0.081\right)$ has a similar total mass as Antlia, while ESO $3060170\left(M_{500} \sim 10^{14} M_{\odot}, z=0.0358\right)$ is slightly more massive.

The entropy profiles are quite similar in the sense that they all rise from the center all the way out to $R_{200}$, and the values at $R_{200}$ are consistent with the gravity heating-only predictions (see also Figure 11). The entropy slope of RX J1159+5531 in the outer regions between $\sim 0.1-1 R_{200}$ is also quite flat, with a power law index of about 0.5 , lower but still consistent with that of $0.69_{-0.24}^{+0.22}$ for Antlia. For ESO 3060170, there are some indications that the entropy near $R_{200}$ drops slightly but not significantly if the ringing effect is corrected, and thus it is consistent with the general trend of Antlia and RX J1159+5531. The density power law index of RX $\mathrm{J} 1159+5531$ is about $\alpha \sim 1.4$, which is flatter than Antlia of $\alpha=1.75_{-0.24}^{+0.27}$. That of ESO 3060170 is much steeper, with $\alpha \approx 2.3$, closer to those of the massive clusters. The gas ( baryon) fractions of all groups are consistent with the cosmic value out to the virial regions, showing no evidence of strong clumping. While both Antlia and ESO 3060170 follow the universal pressure profile, the pressure of RX J1159+5531 is significantly higher beyond $\sim 0.5 R_{500}$, and deviates by more than a factor of two beyond $\sim R_{500}$. Note that the universal pressure profile has a rather small scatter of $\sim 30 \%$ between 0.2 and $1 R_{500}$ for massive clusters and a similar scatter for groups (Arnaud et al. 2010; Sun et al. 2011). Compared to density or entropy, the universal pressure profile is believed to be less sensitive to dynamical history and non-gravitational physics. One might expect that mergers or unrelaxed clusters or groups could have such larger deviations in their pressure profiles, but comparing the dynamically old RX J1159+5531 with large deviations with the dynamically young Antlia shows the opposite trend. Thus, RX J1159+5531 might be an exception.

In summary, we observe a diversity of ICM properties for different low mass groups. Strong disturbances such as cold fronts or sloshing can induce significant deviations from the universal pressure and baseline entropy profiles (e.g., Urban et al. 2014). The dynamically young Antlia is surprisingly relaxed in the sense that it follows the universal pressure profile closely and the entropy profile approaches the gravity heating-only model out to $\sim R_{200}$. Furthermore, no significant fluctuations in temperature and density (or pressure and entropy) profiles were found. The dynamically older cool core Virgo shows evidence of strong heating, perhaps by AGN feedback, which might affect the entropy and pressure out to $\sim R_{500}$. One of the dynamically old fossil groups, ESO 3060170, appears to be relaxed, but the other fossil group RX J1159+5531 surprisingly shows a strong deviation from the universal pressure profile. There is no strong evidence of entropy dropping and also no evidence of clumping in all these systems near the viral regions $\sim R_{200}$, but higher quality data and broader azimuthal coverage is needed to confirm these results for Antlia, RX J1159+5531, and Virgo.

\subsection{Comparison to massive clusters and implications for physics in cluster outskirts}

More than a dozen clusters have been studied with Suzaku in detail out to $\sim R_{200}$ (see a review of Reiprich et al. 2013). Most of these are massive $(T>$ $3 \mathrm{keV}$ ) clusters, and we compare some surprising results found in these massive clusters with Antlia and other groups.

In Figure 10 of Walker et al. (2013), the entropy near $R_{200}$ for massive clusters $\left(M_{500}>\right.$ a few $\left.10^{14} M_{\odot}\right)$ is significantly smaller than the gravity heating-only baseline model, while the others are less clear. For the lowest mass groups, RX J1159+5531 and Virgo in their sample (also the UGC 03957 group recently studied by Thölken et al. 2016), the entropy profiles are consistent with the baseline model near $R_{200}$, similar to Antlia (Section 9.1). Note that the Suzaku results by Walker et al. (2013) are in some tension with the joint ROSAT and Planck data analysis of massive clusters by Eckert et al. (2013), with the latter suggesting a continuously increasing entropy out to $\sim R_{200}$ with only a minor (although still notable) entropy deficit at large radii. Fusco-Femiano \& Lapi (2014) argued that the discrepancy is due to the pressure (instead of temperature) profiles used by Eckert et al. (2013) to determine the entropy, while pressure is insensitive to entropy and therefore temperature should be used instead. If the entropy deficit around $R_{200}$ for massive clusters is real as measured by Walker et al. (2013), this will imply that some non-gravitational processes are responsible for altering the entropy of massive cluster outskirts. For low mass groups where their entropy profiles follow the baseline model at large radii, perhaps nongravitational processes are not important in their outskirts or different non-gravitational effects might cancel out to bring back the final entropy profiles to the baseline model.

Another surprising result is that the measured gas ( baryon) fractions of some massive clusters appear to be significantly higher than the cosmic value (e.g., Simionescu et al. 2011), suggesting biases in the measured gas density (mass) or total mass; although Figure 10 shows that the excess in massive clusters is only marginally significant. However, for the low mass groups discussed in Sections 9.1 there is no evidence for any excess of gas fraction, suggesting that the bias is perhaps minimal on group scales.

One of the explanations for the entropy and gas fraction deviations is the clumping or inhomogeneity in gas (Simionescu et al. 2011), which is expected to be present at some level. The idea is that the emission measure is $\propto n_{e}^{2}$, and therefore we are measuring the average $\left\langle n_{e}^{2}\right\rangle$, which is always higher than $\left\langle n_{e}\right\rangle^{2}$ for clumpy gas. Thus, 
the measured electron density of clumpy gas is biased high and the entropy $\left(K \propto T / n_{e}^{2 / 3}\right)$ is biased low. If the clumps are cool, this can further bias low the entropy. Numerical simulations predict that clumping is stronger for more massive clusters above $10^{14} M_{\odot}$ beyond $\sim R_{200}$ (Nagai \& Lau 2011). However, in these simulations, there appears to be no difference in the clumping level for low or high mass clusters inside the regions of $\sim R_{200}$. This is inconsistent with the observations that more clumping is seen inside $R_{200}$ for more massive clusters. If the low mass groups are indeed as clumpy as the massive clusters inside $R_{200}$ as predicted, this implies that the true average gas density is lower, and hence the true entropy is higher than the baseline model. More theoretical work on the degree of clumpiness in groups is needed to address this issue.

Walker et al. (2013) attempted to separate whether the entropy deficits are due to the deviation of temperature or density from self-similar models. By assuming the self-similar entropy and the universal pressure profiles as the baseline models of the ICM, they suggest that the biases inside $\sim R_{200}$ are primarily due to the bias in density, while beyond that they are due to both biases in temperature and density. In fact, some clusters do not show bias in density but in temperature. Our results of the $K_{200}-T_{500}$ relation also suggest that bias in temperature is needed. This might suggest that different mechanisms are working at different radii and also in different environments. For example, clumping, which may not bias temperature (see the discussion in, e.g., Walker et al. 2013), might start to be important beyond $R_{500}$. Beyond $R_{200}$, perhaps, e.g., non-equipartition electrons and ions might bias low the temperature, and NEI might bias high the electron density and bias low the temperature (Reiprich et al. 2013, see below also). Multiphrase gas might also bias low the temperature. In particular, in Figure 13 of Walker et al. (2013), the temperature is strongly biased low beyond $R_{200}$, which drops to about $0.1-0.2$ of the self-similar model, suggesting the need for some mechanisms that can strongly affect the temperature.

Mass determination using weak lensing has provided important insights to the origins of the gas fraction bias and entropy deficit in some massive clusters (Kawaharada et al. 2010; Ichikawa et al. 2013; Okabe et al. 2014). For Hydra A, Abell 1689, Abell 1835, and Abell 478, where the first three are in the sample of Walker et al. (2013), the HSE masses determined from X-ray are significantly smaller than the weak lensing masses near $R_{200}$. When using the lensing masses instead of the HSE masses to calculate the gas fractions, they approach the cosmic value near $R_{200}$, indicating that the gas fraction bias is mainly due to a bias in the HSE mass rather than a bias in the gas density determination due to clumpy gas. This is consistent with our results on the $f_{\text {gas }, 200}-T_{500}$ relations in Section 8, although the uncertainties of the relations are still quite large. Okabe et al. (2014) also argue that the entropy deficit near $R_{200}$ is primarily due to the steepening in temperature measurements, rather than a shallower density slope. Thus, the joint weak lensing and X-ray studies suggest that the breakdown of HSE is more important than clumping in cluster outskirts (Ichikawa et al. 2013).
Additional pressure support from turbulence or bulk motions (e.g., Lau et al. 2009), higher ion temperature due to non-equipartition, and cosmic rays (e.g., Laganá et al. 2010; Vazza et al. 2012) may be responsible for the nonHSE effects. Weaker accretion shocks have also been suggested to be responsible for the steepening in the outer temperature (Lapi et al. 2010). For groups, there is also some indication that HSE masses are significantly lower than weak leasing masses (Kettula et al. 2013). Thus, energy in addition to those inferred by thermal electrons should be present. Because we do not see an entropy deficit in groups, the total energy injected to the gas might be higher than the pure gravity heating near $R_{200}$. The true gas ( baryon) fraction might in fact be lower than the cosmic value, suggesting that gas might be pushed away by the higher energy injection. It would be interesting to study whether previous AGN feedback can inject enough energy to affect the gas properties out to $R_{200}$ in groups (e.g., Fujita 2001).

As mentioned, non-equilibrium effects due to low density plasma have been considered as another possible mechanism to explain the entropy deficit near $R_{200}$. The electron temperature behind a shock can be lower than the ion temperature, leaving a non-equipartition state of electrons and ions (e.g., Wong \& Sarazin 2009; Avestruz et al. 2015). The ions can also be underionized after a shock, and this NEI plasma has a higher emissivity than the CIE plasma. The soft X-ray emission between 0.3 and $1 \mathrm{keV}$ can be an order of magnitude higher for NEI plasma near the shock region (Wong et al. 2011). One major uncertainty of whether these nonequilibrium processes take effect around $R_{200}$ is the location of accretion shocks where the effects are strongest. By using spherical symmetric hydrodynamic simulations, Wong \& Sarazin (2009) show that the non-equipartition effect is at most a few percent near $R_{200}$ and stronger beyond that, while Avestruz et al. (2015) show that the effect can be up to $\sim 10 \%$ at $\sim R_{200}$ using realistic $3 \mathrm{D}$ simulations Thus, clusters in a realistic environment might induce shocks at smaller radii, making the nonequipartition effect stronger at $R_{200}$.

For a $\sim 10^{15} M_{\odot}$ massive cluster, the NEI effect can bias high the soft X-ray emission by more than $10 \%$ for regions where the non-equipartition effect starts to be important (Wong et al. 2011), and this can bias high the density measured by assuming CIE. The excess soft emission can also bias low the measured temperature (Reiprich et al. 2013). Realistic 3D simulations would be needed to test whether the NEI effect can introduce significant emission bias around $R_{200} 25$ Because the non-equipartition electron temperature is lower than the average temperature of the plasma, the HSE mass estimated using the electron temperature determined by spectral fitting can also be biased low by $\sim 10 \%$ near $R_{200}$ for massive clusters (Avestruz et al. 2015), and thus the gas fraction is further biased high. The 3D simulations also predict that the non-equipartition effect is reduced along filaments, which is qualitatively consistent with the

24 Note that Avestruz et al. (2015) use a scale radius $R_{200 m}$ defined according to the density of matter instead of the critical density, where $R_{200} \approx 0.6 R_{200 m}$.

25 NEI was not included in the simulations by Avestruz et al. (2015). 
higher temperature along the filament directions in Abell 1689 Kawaharada et al. 2010) and Perseus (Urban et al. 2014). Moreover, the density bias is higher along the minor axis of Perseus, which is also qualitatively consistent with the NEI effect being stronger there, but in contrast to the clumping prediction where its effect is more significant along the filament (major) direction. Numerical simulations also suggest that these collisionless effects are more significant for more massive clusters. For groups cooler than $2-3 \mathrm{keV}$, the effects near $R_{200}$ are negligible (Wong \& Sarazin 2009; Wong et al. 2011; Avestruz et al. 2015). Thus, both non-equipartition and NEI effects can potentially explain the entropy deficit and high-gas fraction near $R_{200}$ for massive clusters, and at the same time allow the normal entropy and gas fraction for low mass groups.

With current instruments, it is indeed not easy to distinguish the non-equipartition and NEI effects from other models, such as cooler clumps/subgroups or multitemperature structures, where some or all of them can be working together. Current support mainly comes from timescale estimates, suggesting that these collisionless effects are possible near $R_{200}$. Hoshino et al. (2010) and Akamatsu et al. (2011) estimated that the electronion equipartition timescales could be longer than the shock-elapsed timescales in the massive clusters Abell 1413 and Abell 2142, respectively, suggesting possible non-equipartition of electrons and ions near $R_{200}$. However, they assumed that the shock radii are at $R_{200}$, which is probably an underestimation for virial shocks, and thus might overestimate the significance of the nonequipartition effect. For the massive merger Abell 3667, a shock was detected near $R_{200}$ and the timescale estimations indicate that it is possibly in non-equipartition and NEI (Finoguenov et al. 2010; Akamatsu et al. 2012b). Thus, merging might enhance these non-equilibrium effects near $R_{200}$ as expected. Our estimations for Antlia suggest that the gas around $R_{200}$ is probably in equipartition $\left(T_{e}=T_{i}\right)$. It is also probably in CIE unless $R_{\text {sh }} \lesssim 1.3 R_{200}$. This is consistent with the predictions that these non-equilibrium effects should be small in groups (Wong et al. 2011; Reiprich et al. 2013). A more quantitative study will be needed to test whether or how much these non-equilibrium effects are responsible for the entropy and density biases in massive clusters. In the future, the most direct way to study these effects will be to measure the ion temperature by line width and ionization state by line ratio, which could be possible with the Athena mission (Wong et al. 2011; Nandra et al. 2013).

\section{SUMMARY AND CONCLUSIONS}

We have presented Suzaku observations of the nearest non-cool core cluster, Antlia, out to $1.3 R_{200}$ in the east direction, which is between the major and minor axes of the X-ray emission and also away from the large-scale filament direction. Chandra and XMM-Newton data were used to minimize the point source contamination in all the Suzaku pointings. ROSAT data were also used to ensure the consistency in the soft X-ray background determination. Different systematic uncertainties were taken into account to ensure the results are robust.

The temperature of Antlia drops from about $2 \mathrm{keV}$ near the center down to about $0.7 \mathrm{keV}$ near $R_{200}$, which is consistent with many other clusters. The projected temperature profile of Antlia is consistent with the average scaled profile of other groups and clusters out to $R_{200}$. The power law index of the density profile beyond $\sim 0.1 R_{200}$ is $\alpha=1.75_{-0.24}^{+0.27}$, which is significantly steeper than that of the cool core Virgo Cluster $(\approx 1.2)$, but shallower than those of the massive clusters $(\approx 2-3)$. The pressure of Antlia follows the universal profile out to $\sim R_{200}$.

The entropy profile increases all the way out to $\sim R_{200}$, with its value approaching the gravity heating-only baseline model, but a flatter power law index of $0.69_{-0.24}^{+0.22}$ compared to the baseline model of 1.1. Thus, no entropy deficit is seen near $R_{200}$ as compared to some massive clusters. The entropy inside $R_{500}$ is significantly higher than the baseline model, as has been found in many other groups. Thus, some non-gravitational processes are responsible for the high central entropy.

The gas-mass fraction increases from the center and approaches the cosmic value near $1.3 R_{200}$. Therefore, clumping is not significant in this direction.

The electron-ion equipartition timescale is shorter than the shock-elapsed timescale inside $R_{200}$, suggesting that electrons and ions inside this region should share the same temperature in this low mass group. Although the collisional ionization timescale can be much longer, the plasma in Antlia should still be in CIE near $R_{200}$ unless its shock radius is smaller than about $1.3 R_{200}$.

We compiled X-ray measurements primarily using Suzaku observations in the literature. The $f_{\text {gas }, 200}-T_{500}$ relation has a power law slope of $0.328 \pm 0.166$ for the sample with HSE mass measurements. After correcting for the stellar mass fraction, the enclosed baryon fraction at $R_{200}$ is consistent with the cosmic value.

The power law slope of the $K_{200}-T_{500}$ relation is $0.638 \pm 0.205$, which is significantly smaller than the gravitation heating-only model, and somewhat smaller than the $K_{500}-T_{500}$ relation measured by Sun et al. (2009) or Pratt et al. (2010). The gas corrected $K_{200}-T_{500}$ relation has a larger slope of $0.86 \pm 0.23$. The corrected entropy for massive clusters is still lower than the baseline model. Thus, the entropy deficit at $R_{200}$ is not fully accounted by the bias or deviation in the gas fraction, in contrast to the entropy deficit at smaller radii (Pratt et al. 2010).

We compared the non-cool core Antlia with three other low mass groups, Virgo, ESO 3060170, and RX $\mathrm{J} 1159+5531$, out to $R_{200}$. Counterintuitively, the dynamically youngest Antlia is surprisingly relaxed compared with some other dynamically older groups. Observations in other directions of Antlia are needed to test whether this is due to azimuthal variation. The dynamically older cool core Virgo appears to be strongly heated to a very high entropy out to $\sim R_{500}$, presumably by the strong AGN feedback. While one of the dynamically evolved fossil groups, ESO 3060170, appears to be relaxed, the other fossil group, RX J1159+5531 (with full azimuthal coverage), deviates significantly from the universal pressure profile. Thus, we observe a diversity of ICM properties for different low mass groups.

While massive clusters sometimes show an entropy deficit and an excess in gas fraction near $R_{200}$, there is no such evidence for the lower mass groups we considered. This suggests that clumping and other non-equilibrium processes in low mass groups might not be as significant 
as in the high mass systems. We argued that current data are not sensitive enough to distinguish or address the contributions of different models to explain the entropy and gas fraction deviations in massive cluster outskirts. More observations spanning a wide range of mass and more complete azimuthal coverage, as well as more theoretical efforts, are needed to understand the outskirts of galaxy clusters and groups. A direct detection of clumps and non-equilibrium effects in cluster outskirts may be possible with future missions, such as the SMART-X version of the $X$-ray Surveyor mission concept and the Athena observatory (Vikhlinin et al. 2012; Nandra et al. 2013).
We thank Lucas Johnson, Dacheng Lin, Peter Maksym, Eric Miller, Evan Million, Yuanyuan Su, and Mihoko Yukita for useful discussions. We thank all PIs of the relevant observations and authors of the published works we used for their original efforts. KWW and JAI were supported by NASA ADAP grants NNX13AI52G and NNX13AI53G, as well as Chandra grant GO3-14128A. CLS was partially supported by Chandra grant GO5-16131X and NASA XMM-Newton grant NNX15AG26. YF was supported by KAKENHI No. 15K05080. THR acknowledges support by the Deutsche Forschungsgemeinschaft (DFG) through Heisenberg research grant RE $1462 / 5$ and grant RE $1462 / 6$.

\section{APPENDIX}

\section{A. EMISSION MEASURE AND DENSITY DEPROJECTION}

The left panel of Figure 12 shows the XSPEC APEC normalization per unit surface area of the ICM for the projected spectral analysis, which is proportional to the emission measure of $\int n_{e}^{2} d l$, where $n_{e}$ is the electron density and $l$ is the column length along the line of sight. Systematic uncertainties have been added in quadrature to the statistical uncertainties. The normalization per unit surface area drops from the center out to $\sim R_{200}$. The uncertainty of the last data point is too large to tell whether the emission measure continues to drop beyond $R_{200}$.

We also plot the deprojected APEC normalization for the PROJCT model in the left panel of Figure 12 , and this normalization is proportional to the emission integral $\int n_{e}^{2} d V$, where $V$ is the volume of the full spherical shell assumed in the deprojection. As can be seen in Appendix B. the deprojected normalizations are subject to very large systematic uncertainties in the outer regions, and we can basically only constrain the upper limits of the outer two bins. Nevertheless, the deprojected norms can be used to check for consistency when deriving the electron density using both the projected and deprojected spectral normalizations.

With the projected normalizations (or emission measure) determined in each annulus and assuming spherical symmetry, we can deproject the density profile using the onion peeling method outlined in Kriss et al. (1983) or Wong et al. (2008). In brief, this technique calculates the emission of each spherical shell starting from the outermost annulus toward the center, and the emission measure of each subsequent shell is calculated by subtracting the projected emission measure from the outer shells.

In doing the onion peeling deprojection, ignoring X-ray emission outside the last data bin can bias high the density at the last few data bins, similar to the bias seen in Urban et al. (2011). We corrected for this edge effect by extrapolating the normalization profile out to $150^{\prime}$ using a power law fit to the data between $27^{\prime}$ and $98^{\prime}$. The errors of the extrapolated data were determined from the errors of the power law fit. We made $10^{6}$ simulated emission measure profiles for deprojections. The median of the electron density profile of Antlia is shown in the right panel of Figure 12. The errors were estimated by the $10^{6}$ Monte Carlo simulations.

The deprojected APEC normalizations of the PROJCT model were directly converted to electron density (red circles in the right panel of Figure 12). The uncertainties of the density using the PROJCT model were much larger than the onion peeling method using the projected spectral normalizations; in particular, the outer few data bins are essentially unconstrained. Nevertheless, the inner regions are consistent with one another, suggesting that the results are reliable. We can therefore take the average of the density profiles derived by the two methods using the standard weighted mean $\mu=\sigma \sum x_{i} / \sigma_{i}^{2}$, where the index $i$ indicates the method used, $\sigma=\left(\sum 1 / \sigma_{i}^{2}\right)^{-1}$ is the error on the weighted mean, and $x_{i}$ and $\sigma_{i}$ are the density and its error (90\% confidence region including both statistical and systematic errors) of the associated method, respectively. The average density profile is shown in the upper panel of Figure 7 . This average density profile was used in all the calculations.

\section{B. SYSTEMATIC UNCERTAINTIES}

In Section 2, we characterized the projected spectrum of the ICM component by the APEC model with proper background modeling. To ensure that this characterization of the projected spectra is robust, we check it against systematic uncertainties in spectral modeling introduced by the background modeling, as well as the uncertainty in metallicity as outlined below. All these systematic uncertainties are included in our data analysis.

The systematic uncertainty $\delta_{\text {sys }}$ of each fitted parameter (temperature and gas normalization) is defined as the bestfit parameter minus the nominal best-fit parameter. We calculate the total upper $(u)$ and lower $(l) 90 \%$ confidence errors by adding the systematic and statistic errors in quadrature:

$$
\delta_{\mathrm{tot}, u}^{2}=\sum_{i} \delta_{\mathrm{sys}, i}^{2}+\delta_{\mathrm{stat}, u}^{2} \text { for } \delta_{\mathrm{sys}, i}>0
$$

and 

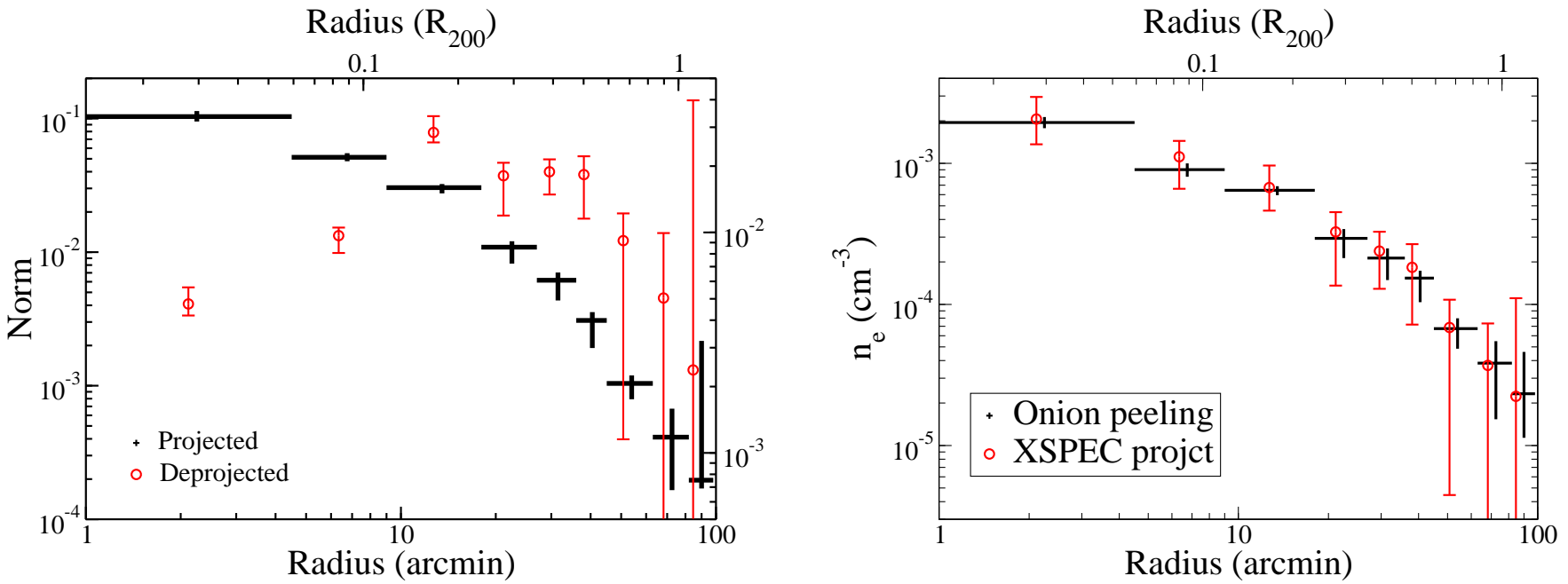

FIG. 12.- Left panel: APEC normalization per unit surface area of the Antlia Cluster for the projected spectral analysis defined as $\frac{20^{2} \pi}{\Omega} \frac{10^{-14}}{4 \pi\left[D_{A}(1+z)\right]^{2}} \int n_{e} n_{\mathrm{H}} d V \mathrm{~cm}^{-5} \operatorname{arcmin}^{-2}$, where $\Omega$ is the solid angle of the source spectral region in units of arcmin ${ }^{2}$ (black crosses corresponding to the left y-axis). Deprojected APEC normalizations for the PROJCT model are also plotted in red circles corresponding to the right $\mathrm{y}$-axis and have been slightly shifted to the left for clarity. The deprojected APEC normalizations are defined as $\frac{10^{-14}}{4 \pi\left[D_{A}(1+z)\right]^{2}} \int n_{e} n_{\mathrm{H}} d V \mathrm{~cm}{ }^{-5}$, with the volume $V$ integrated over the spherical shell of the model. Right panel: electron density profiles of Antlia using the onion peeling (black crosses) and the XSPEC PROJCT (red circles) methods. The red circle data points are slightly shifted to the left for clarity.

$$
\delta_{\mathrm{tot}, l}^{2}=\sum_{i} \delta_{\mathrm{sys}, i}^{2}+\delta_{\mathrm{stat}, l}^{2} \text { for } \delta_{\mathrm{sys}, i}<0,
$$

where $i$ represents each of the systematic uncertainties described below.

\section{NXB uncertainties}

The NXB contributes from less than about $3(10) \%$ of the total $0.6-2.0(0.5-7.0 \mathrm{keV})$ emission at the center up to $37(59) \%$ at the background field (EB). We changed the background level by $\pm 5 \%$. This generally introduces less than $3 \%$ systematic uncertainties in projected temperature within $\sim 30^{\prime}$, but it is larger beyond that (left column in Figure (13). The projected temperature of some of the last data bins can be biased by $\sim 7 \%$, which is smaller than its statistical uncertainty. Similarly, the projected gas normalization is biased by less than $4 \%$ within $\sim 30^{\prime}$. It can be biased high by a factor of five in the outermost bin, which is significantly larger than its statistical uncertainty. The biases to the deprojected quantities are larger compared with the projected quantities, although the statistical uncertainties are also larger. The deprojected temperature within $\sim 30^{\prime}$ can be biased up to $9 \%$ (left column in Figure 15). The bias can be as large as $20 \%$ at the outermost bin, which is comparable to its statistical uncertainty. The systematic uncertainties of the deprojected normalizations within $\sim 45^{\prime}$ are smaller than the statistical uncertainty. For regions beyond that, the deprojected normalizations can be biased by nearly an order of magnitude, highly subject to systematic uncertainties of the NXB.

\section{Galactic absorption uncertainties}

We adopted the Galactic absorption values determined by Willingale et al. (2013), which are generally $\sim 25-30 \%$ higher than those determined by Kalberla et al. (2005). We varied the Galactic values by $\pm 30 \%$ to address these systematic uncertainties. The projected temperature is only biased slightly, up to $\sim 6 \%$ near the center (middle column in Figure 13). The biases in the projected normalizations are similarly small inside $\sim 63^{\prime}$. Beyond that, the bias can be as large as a factor of four. The systematic uncertainty in the deprojected temperature is also generally smaller than $\sim 5-6 \%$, except for the outermost bin that can be biased as large as $\sim 20 \%$ (middle column in Figure 15). The biases in deprojected normalizations are smaller then $\sim 5 \%$ inside $\sim 45^{\prime}$, but again can be biased up to a factor of seven at the outermost bin.

\section{GXB uncertainties}

The temperature of the GXB hot halo was thawed in the spectral fitting, and its best-fit temperature is $T_{\mathrm{HH}} \approx$ $0.5 \mathrm{keV}$. The high temperature may be due to the low Galactic latitude of Antlia. We address the uncertainty of the hot halo temperature by fixing it at a value of $0.3 \mathrm{keV}$, which is more typical for higher Galactic latitude. The low $T_{\mathrm{HH}}$ generally does not change the projected temperature by more than $3 \%$ except at the outer region near $73^{\prime}$, where the bias is about 23\% (right column in Figure 13). Similarly for the projected normalization, the biases are generally smaller than $2 \%$ except for the last data bin, where it can be largely biased by a factor of six. For the deprojected 


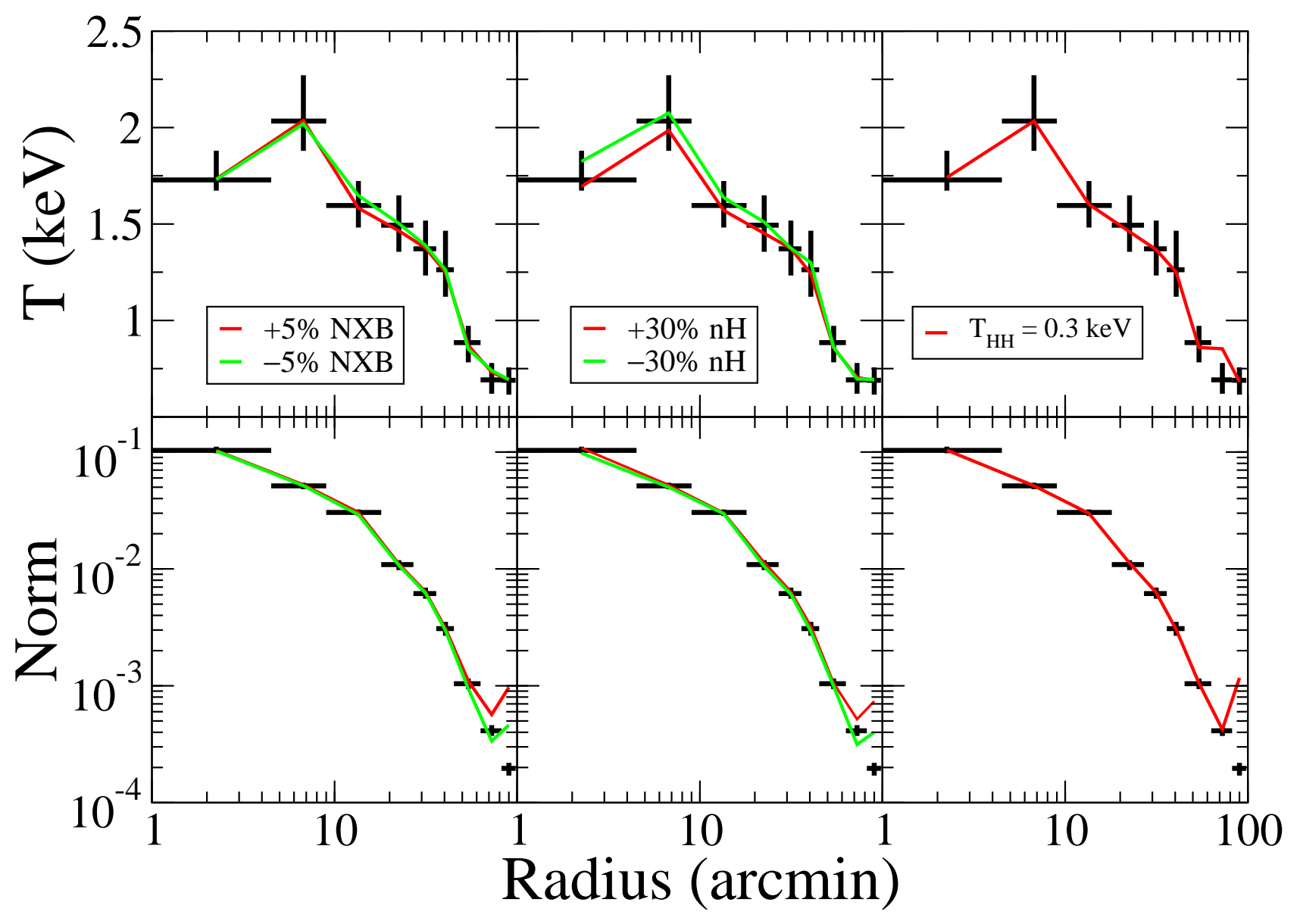

FIG. 13. - Left column: systematic uncertainties introduced by NXB uncertainties. The upper panel shows the temperature profile of the nominal single temperature model (black). Model with NXB contribution increased (decreased) by $5 \%$ is shown in red (green). The lower panel shows the corresponding APEC normalization per unit area. Middle column: similar to left column, but with the red (green) lines representing a model with Galactic absorption $\left(n_{\mathrm{H}}\right)$ increased (decreased) by $30 \%$. Right column: similar to left column, but with the red line representing the model with the Galactic hot halo temperature fixed at $T_{\mathrm{HH}}=0.3 \mathrm{keV}$. For all panels, vertical error bars are at the $90 \%$ statistical confidence level of the nominal model and horizontal bars indicate the radial binning size.

temperature profile, the bias is at most 5\% everywhere (right column in Figure 15). The systematic uncertainties of the deprojected normalizations are smaller than the statistical uncertainties inside $\sim 36^{\prime}$, while they are larger beyond that. The bias can be as large as a factor of six in the outermost bin.

\section{Solar abundance table uncertainties}

Line emission is significant for the low temperature ICM in Antlia, and thus the uncertainty in the solar abundance table might introduce biases in the spectral analysis. We used the aspl solar abundance table for the nominal model, and assessed the systematic uncertainties by using the angr, grsa, and lodd tables (left and middle columns in Figures 14 and 16). The systematic biases in both the projected and deprojected temperatures are all smaller than or comparable to the statistical errors. For the (de-)projected normalizations, the biases are generally less than a few (10) percent within $\sim 45^{\prime}$. Beyond that, the biases increase, and can be as large as a factor of 5 (10) in the outermost bin.

\section{Metallicity uncertainties}

The metallicity of the hot gas beyond $\sim 18^{\prime}$ cannot be constrained with spectral fitting. We fixed it to the lowest metallicity of $0.15 Z_{\odot}$ obtained with the inner region for our nominal model. The metallicity in the cluster outer regions can be as high as its central value (e.g., $0.3 Z Z_{\odot}^{26}$ in Abell 399/401: Fujita et al. 2008; $0.3 Z_{\odot}$ in Perseus: Werner et al.

${ }^{26}$ Converted from the angr to the grsa abundance table. 


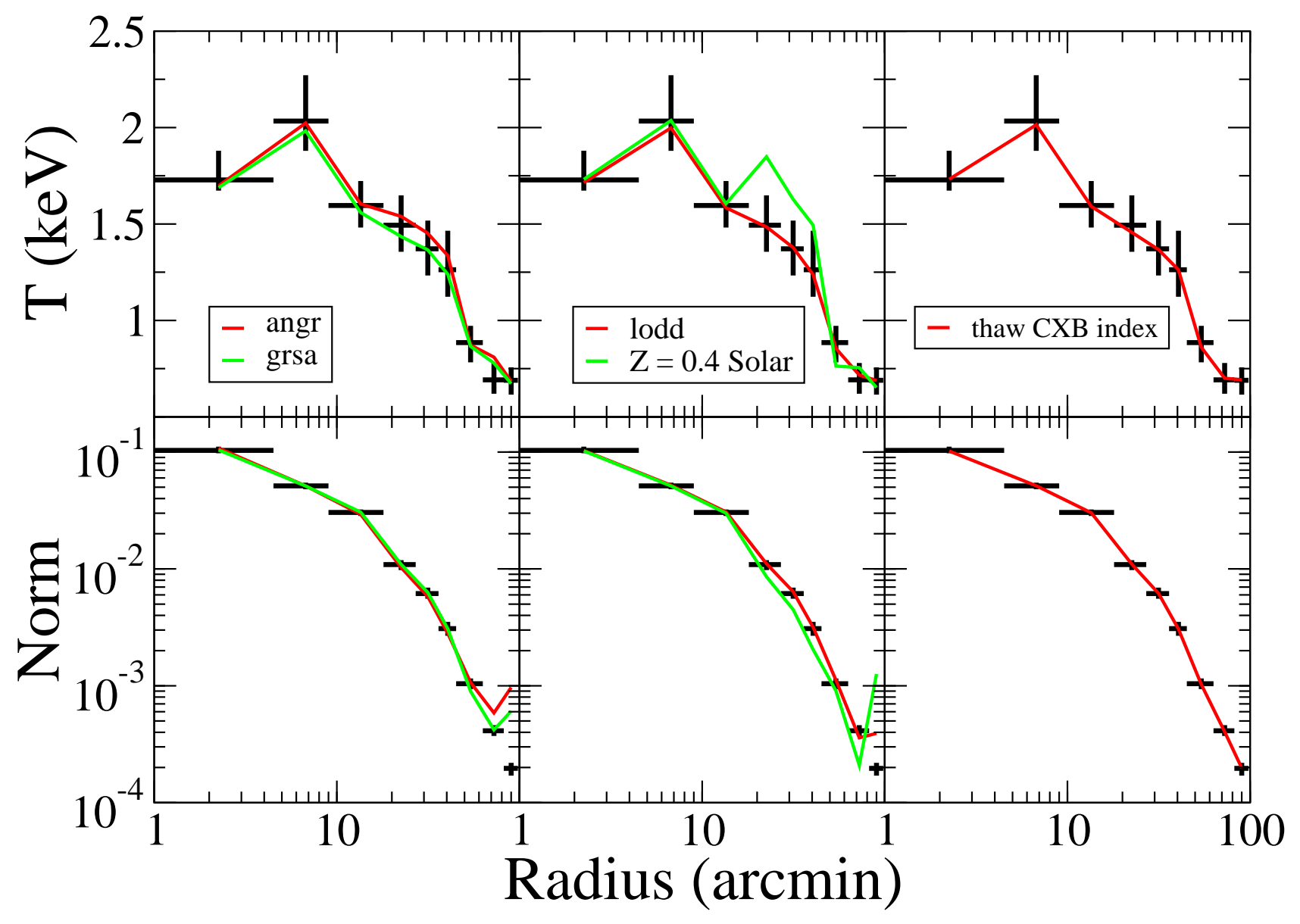

FIG. 14. - Left column: systematic uncertainties introduced by uncertainties in the solar abundance table. The upper panel shows the temperature profile of the nominal single temperature model using the aspl table (black). Model with the angr (grsa) table is shown in red (green). The lower panel shows the corresponding APEC normalization per unit area. Middle column: similar to left column, but with the red line representing a model with the lodd abundance table. The green line shows the model using the aspl table, but the metallicity beyond $27^{\prime}$ is fixed at 0.4 solar. Right column: similar to left column, but with the red line representing model with thawed CXB photon index. For all panels, vertical error bars are at the $90 \%$ statistical confidence level of the nominal model and horizontal bars indicate the radial binning size.

2013). Therefore, we fixed the metallicity of Antlia to $0.4 Z_{\odot}$ beyond $\sim 18^{\prime}$, which is close to its central metallicity. At temperatures below $\sim 1 \mathrm{keV}$, the emission is dominated by line emission that is proportional to metallicity. This generally introduces a degeneracy between metallicity and gas density (because emission is also proportional to density squared). Setting the metallicity to $0.4 Z_{\odot}$ generally decreases the projected and deprojected gas normalizations by up to a factor of two and eight, respectively (middle columns in Figures 14 and 16). However, at the last data bin, both normalizations are biased high by a factor of six, which may be due to the effects coupled with the temperature and background changes. The high metallicity typically introduces a systematic uncertainty in temperature that is comparable to or larger than the statistical uncertainty.

\section{Unresolved CXB uncertainties}

The CXB was modeled with a fixed photon index of 1.4 (Kushino et al. 2002). We assessed the systematic uncertainties by thawing the photon index (right columns in Figures 14 and 16). The best-fit photon index only changes slightly to 1.39 (1.37) for the (de-)projected spectral analysis, introducing systematic uncertainties in temperature that are smaller than the statistical uncertainties. The projected gas normalizations are only affected by $\lesssim 2 \%$. For the deprojected normalizations, the biases are at most up to $\lesssim 10 \%$ and are smaller than the statistical errors inside 


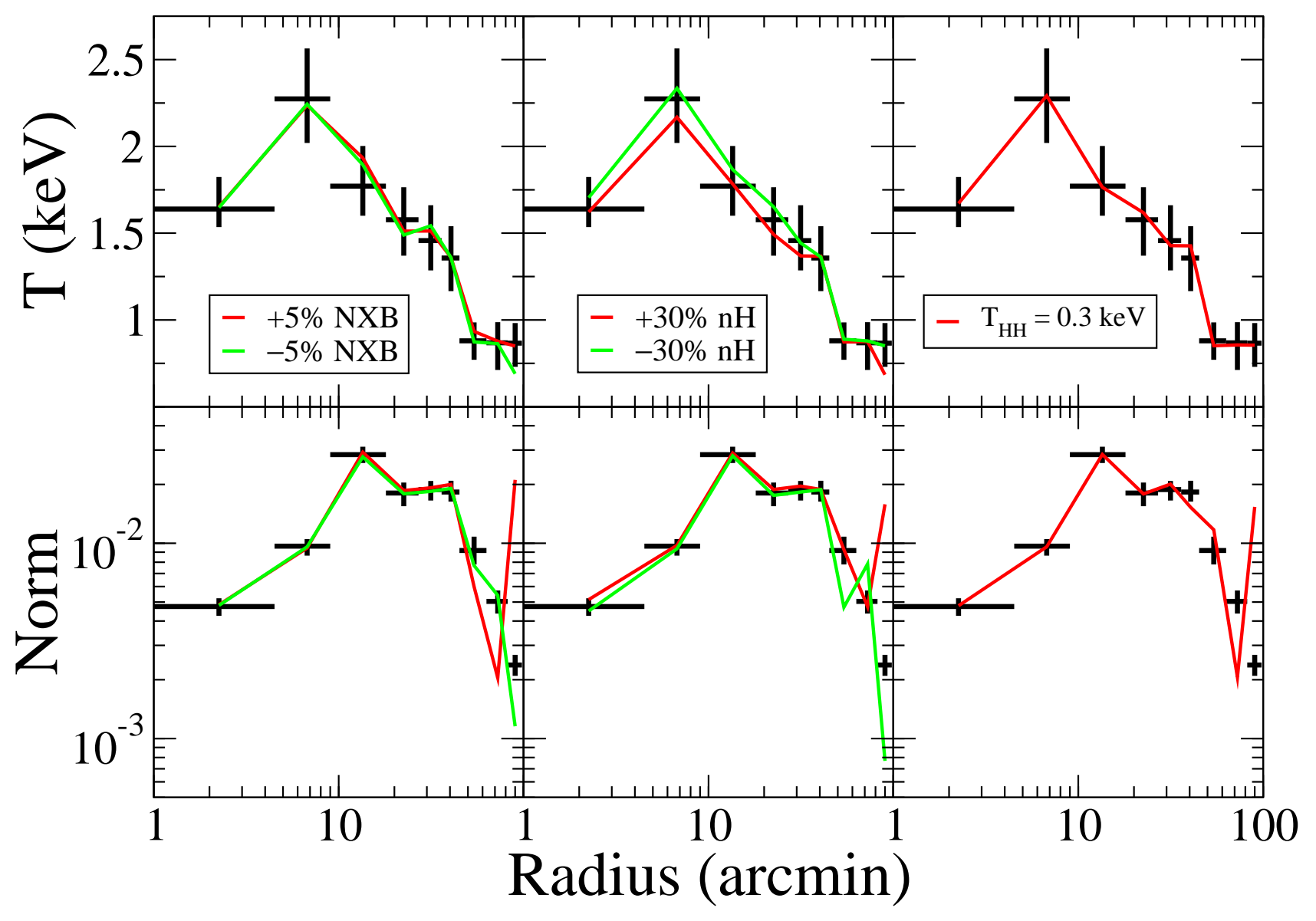

FIG. 15. - Similar to Figure 13 but for the deprojected spectral analysis using the PROJCT model.

$\sim 80^{\prime}$. Beyond that, it can be biased high by a factor of three, which is larger than the statistical uncertainty.

\section{REFERENCES}

Akamatsu, H., Inoue, S., Sato, T., Matsusita, K., Ishisaki, Y., \& Sarazin, C. L. 2013, PASJ, 65, 89

Akamatsu, H., Hoshino, A., Ishisaki, Y., Ohashi, T., Sato, K., Takei, Y., \& Ota, N. 2011, PASJ, 63, 1019

Akamatsu, H., de Plaa, J., Kaastra, J., Ishisaki, Y., Ohashi, T., Kawaharada, M., \& Nakazawa, K. 2012a, PASJ, 64, 49

Akamatsu, H., Takizawa, M., Nakazawa, K., Fukazawa, Y., Ishisaki, Y., \& Ohashi, T. 2012b, PASJ, 64, 67

Akritas, M. G., \& Bershady, M. A. 1996, ApJ, 470, 706

Allen, S. W., Rapetti, D. A., Schmidt, R. W., Ebeling, H., Morris, R. G., \& Fabian, A. C. 2008, MNRAS, 383, 879

Anders, E., \& Grevesse, N. 1989, Geochim. Cosmochim. Acta, 53, 197

Andreon, S. 2010, MNRAS, 407, 263

Arnaud, M., Pratt, G. W., Piffaretti, R., Böhringer, H., Croston, J. H., \& Pointecouteau, E. 2010, A\&A, 517, A92

Asplund, M., Grevesse, N., Sauval, A. J., \& Scott, P. 2009, ARA\&A, 47, 481

Avestruz, C., Nagai, D., Lau, E. T., \& Nelson, K. 2015, ApJ, 808, 176

Bell, E. F., McIntosh, D. H., Katz, N., \& Weinberg, M. D. 2003, ApJS, 149, 289
Bautz, M. W., Miller, E. D., Sanders, J. S., et al. 2009, PASJ, 61, 1117

Bulbul, E., Randall, S. W., Bayliss, M., et al. 2016, ApJ, 818, 131

De Grandi, S., \& Molendi, S. 2002, ApJ, 567, 163

Cantiello, M., Blakeslee, J. P., Raimondo, G., Mei, S., Brocato,

E., \& Capaccioli, M. 2005, ApJ, 634, 239

Eckert, D., Molendi, S., Gastaldello, F., \& Rossetti, M. 2011, A\&A, 529, A133

Eckert, D., Molendi, S., Vazza, F., Ettori, S., \& Paltani, S. 2013, A\&A, 551, A22

Ferguson, H. C., \& Sandage, A. 1990, AJ, 100, 1

Finoguenov, A., Sarazin, C. L., Nakazawa, K., Wik, D. R., \& Clarke, T. E. 2010, ApJ, 715, 1143

Foster, A. R., Ji, L., Smith, R. K., \& Brickhouse, N. S. 2012, ApJ, 756, 128

Fox, D. C., \& Loeb, A. 1997, ApJ, 491, 459

Fujita, Y. 2001, ApJ, 550, L7

Fujita, Y., Tawa, N., Hayashida, K., Takizawa, M., Matsumoto,

H., Okabe, N., \& Reiprich, T. H. 2008, PASJ, 60, 343

Fujita, Y., Ohira, Y., \& Yamazaki, R. 2013, ApJ, 767, L4

Fusco-Femiano, R., \& Lapi, A. 2014, ApJ, 783, 76

Grevesse, N., \& Sauval, A. J. 1998, Space Sci. Rev., 85, 161 


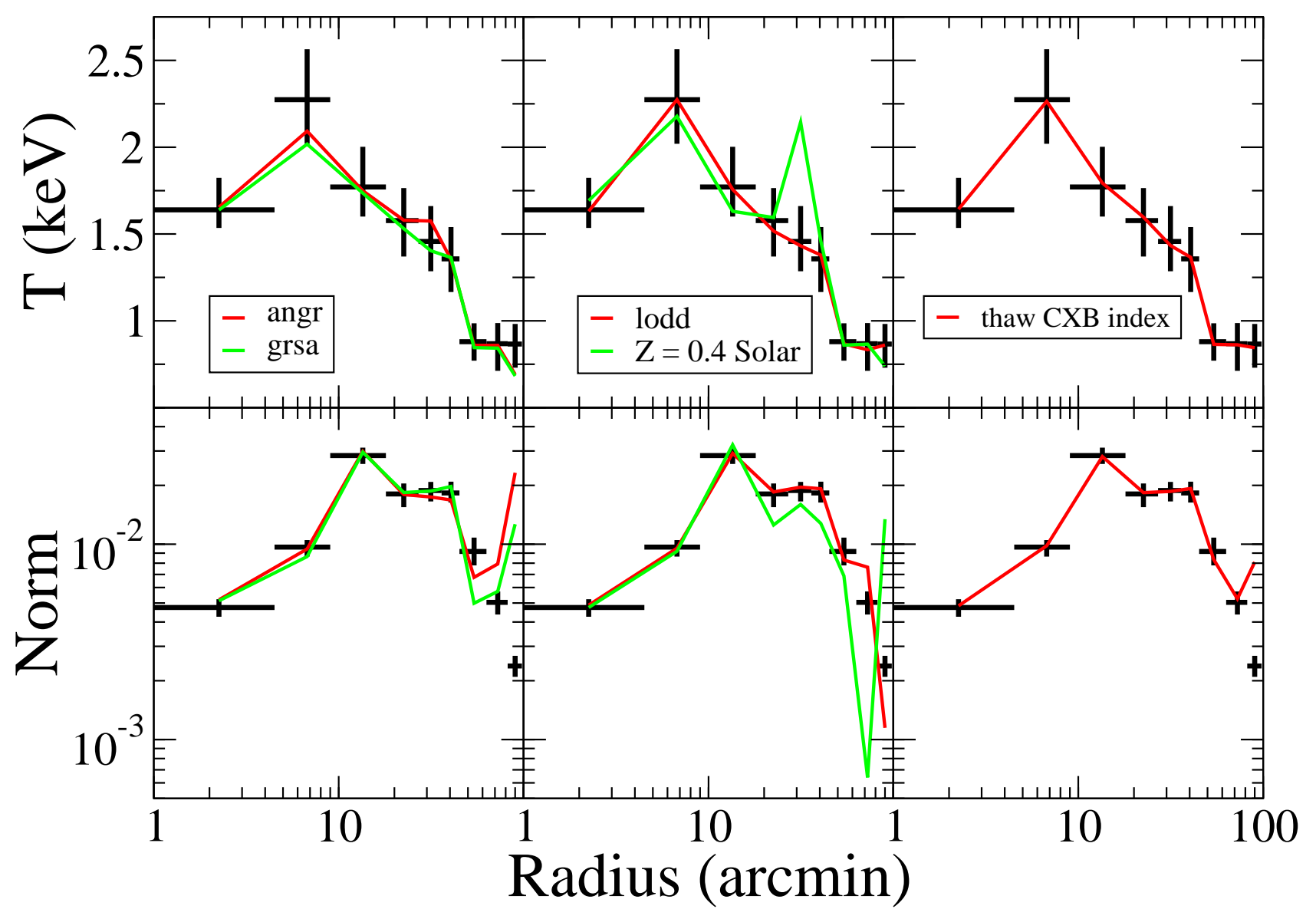

FIG. 16.- Similar to Figure 14 but for the deprojected spectral analysis using the PROJCT model.

Hess, K. M., Jarrett, T. H., Carignan, C., Passmoor, S. S., \& Goedhart, S. 2015, MNRAS, 452, 1617

Hopp, U., \& Materne, J. 1985, A\&AS, 61, 93

Hoshino, A., Henry, J. P., Sato, K., et al. 2010, PASJ, 62, 371

Huchra, J. P., \& Geller, M. J. 1982, ApJ, 257, 423

Humphrey, P. J., Buote, D. A., Brighenti, F., Flohic, H. M. L. G., Gastaldello, F., \& Mathews, W. G. 2012, ApJ, 748, 11

Ibaraki, Y., Ota, N., Akamatsu, H., Zhang, Y.-Y., \& Finoguenov, A. 2014, A\&A, 562, A11

Ichikawa, K., Matsushita, K., Okabe, N. et al. 2013 , ApJ, 766, 90

Ichinohe, Y., Werner, N., Simionescu, A., Allen, S. W., Canning,

R. E. A., Ehlert, S., Mernier, F., \& Takahashi, T. 2015, MNRAS, 448, 2971

Kalberla, P. M. W., Burton, W. B., Hartmann, D., Arnal, E. M., Bajaja, E., Morras, R., \& Pöppel, W. G. L. 2005, A\&A, 440, 775

Kawaharada, M., Okabe, N., Umetsu, K., Takizawa, M., Matsushita, K., Fukazawa, Y., Hamana, T., Miyazaki, S., Nakazawa, K., \& Ohashi, T. 2010, ApJ, 714, 423

Kettula, K., Finoguenov, A., Massey, R., et al. 2013, ApJ, 778, 74

Kriss, G. A., Cioffi, D. F., \& Canizares, C. R. 1983, ApJ, 272, 439

Kushino, A., Ishisaki, Y., Morita, U., Yamasaki, N. Y., Ishida, M., Ohashi, T., \& Ueda, Y. 2002, PASJ, 54, 327

Laganá, T. F., de Souza, R. S., Keller, G. R. 2010, A\&A, 510, A76

Lapi, A., Fusco-Femiano, R., \& Cavaliere, A. 2010, A\&A, 516, A34
Lau, E. T., Kravtsov, A. V., \& Nagai, D. 2009, ApJ, 705, 1129

Lin, Y.-T., Mohr, J. J., \& Stanford, S. A. 2004, ApJ, 610,745

Lodders, K. 2003, ApJ, 591, 1220

Lovisari, L., Reiprich, T. H., \& Schellenberger, G. 2015, A\&A, 573, A118

Mahdavi, A., Hoekstra, H., Babul, A., Bildfell, C., Jeltema, T., \& Henry, J. P. 2013, ApJ, 767, 116

Miller, E. D., Bautz, M., George, J., et al. 2012, in AIP Conf. Ser. 1427 (Melville, NY: AIP), 13

Morandi, A., Sun, M., Forman, W., \& Jones, C. 2015, MNRAS, 450, 2261

Moretti, A., Campana, S., Lazzati, D., \& Tagliaferri, G. ApJ, 588,696

Moretti, A., Pagani, C., Cusumano, G., et al. 2009, A\&A, 493,501

Nakazawa, K., Makishima, K., Fukazawa, Y., \& Tamura, T. 2000, PASJ, 52, 623

Nagai, D., \& Lau, E. T. 2011, ApJ, 731, L10

Nandra, K., Barret, D., Barcons, X., et al. 2013, arXiv:1306.2307

Okabe, N., Umetsu, K., Tamura, T., et al. 2014, PASJ, 66, 99

Pedersen, K., Yoshii, Y., \& Sommer-Larsen, J. 1997, ApJ, 485, L17

Planck Collaboration, Ade, P. A. R., Aghanim, N., et al. 2014, A\&A, 571, A16

Pratt, G. W., Arnaud, M., Piffaretti, R., et al. 2010, A\&A, 511, A85 
Reiprich, T. H., Basu, K., Ettori, S., Israel, H., Lovisari, L., Molendi, S., Pointecouteau, E.\& Roncarelli, M. 2013, Space Sci. Rev., 177, 195

Reiprich, T. H. and Böhringer, H. 2002, ApJ, 567, 716

Sarazin, C. L. 1986, Reviews of Modern Physics, 58, 1

Sato, K., Matsushita, K., Yamasaki, N. Y., Sasaki, S., \& Ohashi, T. 2014, PASJ, 66, 85

Sato, T., Sasaki, T., Matsushita, K., et al. 2012, PASJ, 64, 95

Simionescu, A., Allen, S. W., Mantz, A., et al. 2011, Science, 331 , 1576

Simionescu, A., Werner, N., Urban, O. et al. 2013, ApJ, 775, 4

Simionescu, A., Werner, N., Urban, O., Allen, S. W., Ichinohe, Y., \& Zhuravleva, I. 2015, ApJ, 811, L25

Smith, R. K., Brickhouse, N. S., Liedahl, D. A., \& Raymond, J. C. 2001, ApJ, 556, L91

Smith, R. K., \& Hughes, J. P. 2010, ApJ, 718, 583

Smith Castelli, A. V., Bassino, L. P., Richtler, T., Cellone, S. A., Aruta, C., \& Infante, L. 2008, MNRAS, 386, 2311

Spitzer, L. 1962, Physics of Fully Ionized Gases (2nd ed.; New York: Interscience), 135

Su, Y., White, III, R. E., \& Miller, E. D. 2013, ApJ, 775, 89

Su, Y., Buote, D., Gastaldello, F., \& Brighenti, F. 2015, ApJ, 805,104

Sun, M. 2012, NJPh, 14, 045004

Sun, M., Voit, G. M., Donahue, M., Jones, C., Forman, W., \& Vikhlinin, A. 2009, ApJ, 693, 1142

Sun, M., Sehgal, N., Voit, G. M., Donahue, M., Jones, C., Forman, W., Vikhlinin, A., \& Sarazin, C. 2011, ApJ, 727, L49

Takizawa, M. 1998, ApJ, 509, 579

Tawa, N., Hayashida, K., Nagai, M. 2008, PASJ, 60, 11

Thölken, S., Lovisari, L., Reiprich, T. H., \& Hasenbusch, J. 2016 , A\&A, in press (arXiv:1603.05255)

Trac, H., Bode, P., \& Ostriker, J. P. 2011, ApJ, 727, 94
Urban, O., Werner, N., Simionescu, A., Allen, S. W., \& Böhringer, H. 2011, MNRAS, 414, 2101

Urban, O., Simionescu, A., Werner, et al. 2014, MNRAS, 437, 3939

Vazza, F., Brüggen, M., Gheller, C., \& Brunetti, G. 2012, MNRAS, 421, 3375

Vikhlinin, A., Burenin, R. A., Ebeling, H., et al. 2009, ApJ, 692, 1033

Vikhlinin, A., Reid, P., Tananbaum, H., et al. 2012, Proc. SPIE, 8443,844316

Voit, G. M., Kay, S. T., \& Bryan, G. L. 2005, MNRAS, 364, 909

Walker, S. A., Fabian, A. C., Sanders, J. S., George, M. R., \& Tawara, Y. 2012a, MNRAS, 422, 3503

Walker, S. A., Fabian, A. C., Sanders, J. S., \& George, M. R. 2012b, MNRAS, 424, 1826

Walker, S. A., Fabian, A. C., Sanders, J. S., \& George, M. R. 2012c, MNRAS, 427, L45

Walker, S. A., Fabian, A. C., Sanders, J. S., Simionescu, A., \&

Tawara, Y. 2013, MNRAS, 432, 554

Werner, N., Urban, O., Simionescu, A., \& Allen, S. W. 2013, Nature, 502, 656

Willingale, R., Starling, R. L. C., Beardmore, A. P., Tanvir, N. R., \& O'Brien, P. T. 2013, MNRAS, 431, 394

Wong, K.-W., \& Sarazin, C. L. 2009, ApJ, 707, 1141

Wong, K.-W., Sarazin, C. L., Blanton, E. L., \& Reiprich, T. H. 2008 ApJ, 682, 155

Wong, K.-W., Sarazin, C. L., \& Ji, L. 2011, ApJ, 727, 126

Wong, K.-W., Sarazin, C. L., \& Wik, D. R. 2010, ApJ, 719, 1

Yang, H.-Y. K., Ricker, P. M., \& Sutter, P. M. 2009, ApJ, 699, 315

Yoshino, T., Mitsuda, K., Yamasaki, N. Y., et al. 2009, PASJ, 61, 805 